\title{
Identification of key genes and biological pathways in Chinese lung cancer population using bioinformatics analysis
}

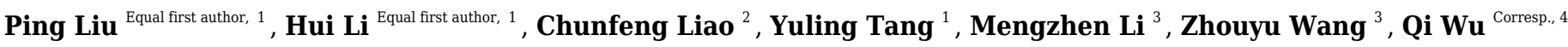 \\ , Yun Zhou Corresp. 5 \\ 1 Department of Respiratory Medicine, The first hospital of Changsha, Changsha, China \\ 2 Department of Cardiology, The first hospital of Changsha, Changsha, China \\ 3 MyGene Diagnostics Co., Ltd, Guangzhou, China \\ 4 Department of Emergency, The first hospital of Changsha, Changsha, China \\ 5 Department of Spinal Surgery, The first hospital of Changsha, Changsha, China \\ Corresponding Authors: Qi Wu, Yun Zhou \\ Email address: 392554098@qq.com, zhouyun83@sina.com
}

Background: Identification of accurate prognostic biomarkers is still particularly urgent for improving the poor survival of lung cancer patients. In this study, we aimed to identity the potential biomarkers in Chinese lung cancer population via bioinformatics analysis. Methods: In this study, the differentially expressed genes (DEGs) in lung cancer were identified using 6 datasets from Gene Expression Omnibus (GEO) database. Subsequently, enrichment analysis was conducted to evaluate the underlying molecular mechanisms involved in progression of lung cancer. Protein-protein interaction (PPI) and CytoHubba analysis were performed to determine the hub genes. The GEPIA, Human Protein Atlas (HPA), Kaplan-Meier plotter, and TIMER databases were used to explore the hub genes. The receiver operating characteristic (ROC) analysis was performed to evaluate the diagnostic value of hub genes. Reverse transcription quantitative PCR (qRT-PCR) was used to validate the expression levels of hub genes in 10 pairs of lung cancer paired tissues. Results: A total of 499 overlapping DEGs (160 upregulated and 339 downregulated genes) were identified in the microarray datasets. DEGs were mainly associated with pathways in cancer, focal adhesion, and protein digestion and absorption. There were 9 hub genes (CDKN3, MKI67, CEP55, SPAG5, AURKA, TOP2A, UBE2C, CHEK1 and BIRC5) identified by PPI and module analysis. In GEPIA database, the expression levels of these genes in lung cancer tissues were significantly upregulated compared with normal lung tissues. The results of prognostic analysis showed that relatively higher expression of hub genes was associated with poor prognosis of lung cancer. In HPA database, most hub genes were highly expressed in lung cancer tissues. The hub genes have good diagnostic efficiency in lung cancer and normal tissues. The expression of any hub gene was associated with the infiltration of at least two immune cells. qRT-PCR confirmed that the 
expression level of CDKN3, MKI67, CEP55, SPAG5, AURKA, TOP2A were highly expressed in lung cancer tissues. Conclusions: The hub genes and functional pathways identified in this study may contribute to understand the molecular mechanisms of lung cancer. Our findings may provide new therapeutic targets for lung cancer patients. 
2

3 Running title: Identification of candidate genes in lung cancer

\section{Authors and affiliations:}

6 Ping Liu ${ }^{1 *}$, Hui Li ${ }^{1 *}$, Chunfeng Liao ${ }^{2}$, Yuling Tang ${ }^{1}$, Mengzhen Li $^{3}$, Zhouyu Wang ${ }^{3}, \mathrm{Qi} \mathrm{Wu}^{4}, \mathrm{Yun}_{\mathrm{Zhou}}^{5}$

$7{ }^{1}$ Department of Respiratory Medicine, The first hospital of Changsha, Changsha 410005, China

$8{ }^{2}$ Department of Cardiology, The first hospital of Changsha, Changsha 410005, China

$9 \quad$ 3MyGene Diagnostics Co., Ltd, Guangzhou 510000, China

${ }^{4}$ Department of Emergency, The first hospital of Changsha, Changsha 41000, China

${ }^{5}$ Department of Spinal Surgery, The first hospital of Changsha, Changsha 410005, China

\section{Correspondence to:}

\section{Qi Wu}

Department of Emergency, The first hospital of Changsha, 311 Yingpan Road, Kaifu District, Changsha 410005,

15 China

Tel: +86-0731-84667562

Email: 392554098@qq.com

\section{And Yun Zhou}

Department of Spinal Surgery, The first hospital of Changsha, 311 Yingpan Road, Kaifu District, Changsha 410005, China

Tel: $+86-0731-84667562$

Email: zhouyun83@sina.com

\section{"These authors contributed equally to this work.}




\section{Abstract}

Background: Identification of accurate prognostic biomarkers is still particularly urgent for improving the poor survival of lung cancer patients. In this study, we aimed to identity the potential biomarkers in Chinese lung cancer population via bioinformatics analysis.

Methods: In this study, the differentially expressed genes (DEGs) in lung cancer were identified using 6 datasets from Gene Expression Omnibus (GEO) database. Subsequently, enrichment analysis was conducted to evaluate the underlying molecular mechanisms involved in progression of lung cancer. Protein-protein interaction (PPI) and CytoHubba analysis were performed to determine the hub genes. The GEPIA, Human Protein Atlas (HPA), Kaplan-Meier plotter, and TIMER databases were used to explore the hub genes. The receiver operating characteristic (ROC) analysis was performed to evaluate the diagnostic value of hub genes. Reverse transcription quantitative PCR (qRT-PCR) was used to validate the expression levels of hub genes in 10 pairs of lung cancer paired tissues.

Results: A total of 499 overlapping DEGs (160 upregulated and 339 downregulated genes) were identified in the microarray datasets. DEGs were mainly associated with pathways in cancer, focal adhesion, and protein digestion and absorption. There were 9 hub genes (CDKN3, MKI67, CEP55, SPAG5, AURKA, TOP2A, UBE2C, CHEK1 and BIRC5) identified by PPI and module analysis. In GEPIA database, the expression levels of these genes in lung cancer tissues were significantly upregulated compared with normal lung tissues. The results of prognostic analysis showed that relatively higher expression of hub genes was associated with poor prognosis of good diagnostic efficiency in lung cancer and normal tissues. The expression of any hub gene was associated with the infiltration of at least two immune cells. qRT-PCR confirmed that the expression level of CDKN3, MKI67, CEP55, SPAG5, AURKA, TOP2A were highly expressed in lung cancer tissues.

Conclusions: The hub genes and functional pathways identified in this study may contribute to understand the molecular mechanisms of lung cancer. Our findings may provide new therapeutic targets for lung cancer patients.

Keywords: lung cancer, Chinese population, hub genes, therapeutic targets 


\section{Introduction}

Lung cancer has become the most common type of cancer in the world, leading to the largest number of cancer-related deaths (Siegel et al.,2019). More than 80\% of lung cancer are non-small cell lung cancer (NSCLC), mainly lung adenocarcinoma (LUAD) and lung squamous cell carcinoma (LUSC) (Travis,2011). LUAD accounts for over 70\% of NSCLC (Hirsch et al.,2016). Smoking is the most important risk factor for lung cancer, many other lifestyle and occupational factors also have a significant impact (Parida et al.,2021). Changes in risk factors for cancer, especially diet, obesity, diabetes, and air pollution, continue to fuel the trend of cancer transformation in China (Sun et al.,2020). Although there are various treatment methods for lung cancer, including surgery, chemotherapy, radiotherapy, targeted therapy, immunotherapy, and palliative treatment, the 5-year survival rate of lung cancer in recent decades is still very low (Wang et al.,2020). Therefore, the identification of accurate prognostic biomarkers and novel therapeutic targets is still particularly urgent for improving the poor survival of NSCLC patients.

In recent years, with the development of microarray and high-throughput sequencing technologies, a large number of open data resources, such as the Cancer Genome Atlas Database (TCGA) and Gene Expression Comprehensive Database (GEO), have generated a large amount of gene data $(Y u$ and Tian,2020). Bioinformatics can effectively screen and mine microarray data, thereby revealing potential oncogenes at the molecular level (Gu et al.,2018; Liu et al.,2019). Bioinformatics has been widely used to find molecular markers and signaling pathways related to the occurrence and development of lung cancer (Jiao et al.,2020;Li et al.,2020). Possibly driven by the community and environmental factors, the observed differences in disease incidence suggest the importance of residential location in risk assessment of lung cancer (Zhu et al.,2020). At present, most of the GEO datasets used for lung cancer research are from different countries (Song et al.,2021). In this study, the differentially expressed genes (DEGs) in lung cancer were identified using 6 GEO datasets from Chinese population. Then, functional enrichment analysis was conducted to evaluate the underlying molecular mechanisms involved in progression of lung cancer. Subsequently, we conducted protein-protein 
82

83

84

GEPIA, Human Protein Atlas (HPA), Kaplan-Meier plotter, and TIMER databases were used to explore the hub genes. ROC analysis was performed to evaluate the diagnostic value of hub genes. Finally, Reverse transcription quantitative PCR (qRT-PCR) was used to validate the expression levels of hub genes in 10 pairs of lung cancer paired tissues. Our research will provide some useful biomarkers for the diagnosis and prognosis of lung cancer.

\section{Materials and Methods}

\section{The information of GEO datasets}

Six datasets, including GSE136043, GSE130779, GSE118370, GSE85841, GSE85716, and GSE89039 were selected from GEO database (https://www.ncbi.nlm.nih.gov/geo/). The inclusion criteria for the above datasets were set as follows: (1) The samples of the datasets were all from China; (2) the datasets included human lung cancer tissues and normal tissues; (3) the number of samples in each dataset was more than 10. The GSE136043 dataset included 5 LUAD samples and 5 normal samples. The GSE130779 dataset included 8 LUAD samples and 8 normal samples. The GSE118370 dataset included 6 LUAD samples and 6 normal samples. The GSE85841 dataset included 8 LUAD samples and 8 normal samples. The GSE85716 dataset included 6 LUAD samples and 6 normal samples. The GSE89039 dataset included 8 LUAD samples and 8 normal samples. Six datasets included a total of 41 LUAD tissues and 41 normal lung tissues (Table 1).

\section{Identification of DEGs}

$\mathrm{R}$ packages (GEOquery and dplyr) were performed to match the expression matrix to the probe ( $L i$ et al.,2020). The DEGs in each microarray were filtrated by the limma package. RobustRankAggreg (RRA) was used to integrate the DEGs identified from six datasets (Kolde et al.,2012). The RRA algorithm can handle a variable number of genes identified from different microarray platforms. Next, $|\log 2 \mathrm{FC}|>1.0$ and adjusted $P$ value $<0.05$ were used to filtrate the DEGs.

\section{GO and KEGG enrichment analysis}

The DAVID database (v6.8, https://david.ncifcrf.gov/) was used to perform the Gene Ontology (GO) and Kyoto Encyclopedia of Genes and Genomes (KEGG) enrichment analysis (Huang et al.,2009). The results of 
GO annotation contain three parts, including biological process (BP), cell component (CC), and molecular function (MF). The top 15 GO terms were listed according to P-value. The results were considered statistically significant if $P<0.05$. The KEGG pathways were visualized by ggplot2 package $(P<0.05)$ (Tang et al.,2020).

\section{PPI network construction and hub genes identification}

The protein-protein interactions of the overlapping DEGs were obtained via the STRING database (https://string-db.org/) (Szklarczyk et al.,2019). The combined score of medium confidence $>0.4$ was used as the cut-off value in the STRING database. Subsequently, a clear illustration of the PPI was demonstrated using Cytoscape software (v3.8.0) with CytoHubba, which is a plug-in that uses the degree algorithm to screen the hub genes (Ma et al.,2020). The degree, edge percolated component (EPC), maximal clique centrality algorithm (MCC), and maximum neighborhood component (MNC) algorithms in CytoHubba were used to select the hub genes (Ma et al.,2021). The top 20 nodes with the degree, EPC, MCC and MNC were selected, and we take the intersection of the four algorithm as the hub genes. The Cytoscape plug-in Molecular Complex Detection $($ MCODE $)($ degree cutoff $=2$, Node Score Cutoff $=0.2$, and $\mathrm{K}-$ Core $=2)$ was used to capture the hub network modules (Dai et al.,2020).

\section{Validation of mRNA expression levels of hub genes}

The GEPIA (http://gepia.cancer-pku.cn/index.html) is an online database that consists of 9736 tumors and 8587 normal samples from TCGA and GTEx data (Mou et al.,2021). The mRNA expression levels of hub genes were validated by GEPIA database.

\section{Validation of hub genes via Kaplan Meier plotter database}

The identification of overall survival (OS) rates of hub genes in LUAD was performed using the Kaplan Meier plotter database (https://kmplot.com/analysis), an online tool used to assess the effect of $54 \mathrm{k}$ genes on survival across 21 cancer types (Liu et al.,2020). The Kaplan-Meier plotter is an online deposit of the survival analysis data of EGA, TCGA, and GEO (Affymetrix microarrays only) databases (Yang et al.,2020). A log-rank $P<0.05$ was considered to be statistically significant. 
137

\section{Validation of protein expression levels of hub genes in HPA database}

The protein expression levels of hub genes in lung cancer tissues and normal tissues were validated using immunohistochemistry (IHC) results from the HPA database (https://www.proteinatlas.org/) (Li et al.,2020).

\section{Validation of hub genes by ROC analysis}

Then, we performed a receiver operating characteristic (ROC) analysis using TCGA database to evaluate the diagnostic value of hub genes (Jia et al.,2021). Usually, the AUC value $>0.85$ showed a good diagnostic value for lung cancer.

\section{Immune infiltrates analysis of hub genes}

Tumor immune estimation resource (TIMER) (https://cistrome.shinyapps.io/timer/) is a comprehensive website for systematic analysis of tumor infiltrating immune cells of 32 different cancers in TCGA database(Yang et al.,2021). In this study, TIMER database was used to estimate the associations between hub genes expression and immune cell populations (B cells, CD8 + T cells, CD4 + T cells, macrophages, neutrophils and dendritic cells) in LUAD.

\section{Lung cancer tissues}

Tumor and adjacent normal tissues were obtained from 10 lung cancer patients in the The First Hospital of Changsha between September 2021 and October 2021. These patients had no other major illnesses. A total of 20 frozen tissue specimens contained 10 tumor tissues and 10 matched adjacent non-tumor tissues were obtained. The detailed clinical information of the patients is shown in Table S1. All tissues were preserved and stored at $-80^{\circ} \mathrm{C}$. The study was approved by the Ethics Committee of The First Hospital of Changsha and informed consent was obtained from all patients.

\section{Quantitative real-time reverse transcription PCR (qRT-PCR)}

Total RNA from lung cancer patients' tissues was isolated by TRIzol reagent (Invitrogen, Carlsbad, CA, 
163

164

165

166

167

168

169

170

171

172

173

174

175

176

177

178

179

180

181

182

183

184

185

186

187

188

189

USA). Real-time PCR was further performed with SYBR Green Master Mix (Takara, Japan) according to the manufacturer's protocols. The primers used in this study are provided in Table S2. GAPDH were used as an internal control and $2^{-\Delta \Delta \mathrm{Ct}}$ method was applied to evaluate gene expression levels. Differences in relative expression levels were analysed through $\mathrm{t}$ test or M ann-Whitney U test (SPSS, V 22.0). The results were considered statistically significant if $P<0.05$.

\section{Results}

\section{Identification of DEGs among four GEO datasets}

The LUAD chip expression datasets GSE136043, GSE130779, GSE118370, and GSE85841, GSE85716, and GSE89039 were normalized（Fig. S1）. The GSE136043 contained 1206 DEGs, including 541 upregulated genes and 665 downregulated genes. The GSE130779 dataset contained 2964 DEGs, including 1231 upregulated genes and 1733 downregulated genes. The GSE118370 dataset contained 789 DEGs, including 211 upregulated genes and 578 downregulated genes. The GSE85841 dataset contained 3058 DEGs, including 1270 upregulated genes and 1788 downregulated genes. The GSE85716 dataset contained 1196 DEGs, including 367 upregulated genes and 829 downregulated genes. The GSE89039 dataset contained 2966 DEGs, including 1129 upregulated genes and 1837 downregulated genes. The volcano plots of DEGs in the six datasets are shown in Fig. 1. A total of 499 DEGs were obtained through the RRA method, including 160 upregulated genes (Table S3) and 339 downregulated genes (Table S4). The top 20 up- and down-regulated genes after the integrated analysis are shown in Fig. 2.

\section{Functional enrichment analysis of overlapping DEGs}

The 499 overlapping DEGs were subjected to the GO and KEGG enrichment analysis. The top 15 enriched GO terms from biological process, cellular component, and molecular function are shown in Fig. 3A. In the biological process, the DEGs were mainly enriched in angiogenesis, collagen catabolic process, and cell adhesion. In the cellular component, the DEGs were mainly enriched in extracellular region, proteinaceous extracellular matrix, and extracellular space. In the molecular function, the DEGs were mainly enriched in heparin binding, calcium ion binding, and metalloendopeptidase activity. For the KEGG pathways analysis, the 
190

191

192

193

194

195

196

197

198

199

200

201

202

203

204

205

206

207

208

209

210

211

212

213

214

215

216

DEGs were mainly enriched in pathways in cancer, focal adhesion, and protein digestion and absorption (Fig. 3B).

\section{PPI network construction and hub genes identification}

The PPI network included 423 nodes and 1331 edges (Fig. 4A). We interacted the results of four algorithms to improve the reliability of the hub genes (Table 2). A total of nine genes (CDKN3, MKI67, CEP55, SPAG5, AURKA, TOP2A, UBE2C, CHEK1 and BIRC5) were considered hub genes. The top three modules from MCODE were selected for future analysis. Module 1 included 21 upregulated genes, including TOP2A, INCENP, UBE2C, BIRC5, AURKA, TRIP13, CENPM, MND1, RAD54L, STIL, CHEK1, KIF14, GTSE1, CDC25C, MKI67, CEP55, MELK, CDKN3, SPAG5, CENPF, KIF20A (Fig. 4B). Module 2 included 14 upregulated genes and 5 downregulated genes (Fig. 4C). Module 3 included 1 upregulated gene and 5 downregulated genes (Fig.4D). The functional enrichment analysis of genes in module 1 were conducted by DAVID. These genes were significantly enriched in cell division, midbody and ATP binding (Table 3).

\section{Validation of hub genes by GEPIA and Kaplan Meier plotter database}

We validated mRNA expression levels of hub genes in the LUAD cohorts from GEPIA database. The LUAD cohort included 483 tumor tissues and 347 normal tissues. The mRNA expression levels of hub genes were higher in LUAD tissues than in normal lung tissues (Fig. 5). Results from the Kaplan Meier plotter revealed that relatively higher expression of hub genes was associated with poor prognosis of LUAD patients (Fig. 6).

\section{Protein expression levels of hub genes in HPA database}

The protein expression levels of hub genes were explored using the HPA database. As the immunohistochemical information of $C D K N 3$ and $C H E K 1$ were not existed in HPA, we have only displayed the results of MKI67, CEP55, SPAG5, AURKA, TOP2A, UBE2C and BIRC5 (Fig. 7). The protein levels of MKI67, $A U R K A$ and BIRC5 were not detected in normal lung tissues, while the levels of these genes were high in lung cancer tissues. No expression of CEP55 was observed in normal lung tissues, while medium expression of CEP55 was observed in tumor tissues. The protein levels of $T O P 2 A$ and $U B E 2 C$ were low in normal lung tissues, 
217

218

219

220

221

222

223

224

225

226

227

228

229

230

231

232

233

234

235

236

237

238

239

240

241

242

243

while the levels of these genes were high in lung cancer tissues. SPAG5 was found to have medium expression in LUAD tissues, while low expression was observed in normal lung tissues. The results of HPA database showed that most hub genes may be highly expressed in lung cancer tissues.

\section{The diagnostic value of hub genes in LUAD and their relationships with tumor infiltrating immune cells}

The ROC curve was used to evaluate the diagnostic value of hub genes. As shown in Fig. 8 , the AUC values of CDKN3, MKI67, CEP55, SPAG5, AURKA, TOP2A, UBE2C, CHEK1 and BIRC5 in LUAD were 0.965, 0.965, $0.980,0.986,0.975,0.986,0.984,0.978,0.980$, respectively. Thus, the hub genes have good diagnostic efficiency in LUAD and normal tissues. The results from TIMER database showed that the 9 hub genes were not associated with tumor purity (Figure 9). However, the expression of these genes was negatively correlated with B cell infiltration $(P<0.05)$. Only the TOP $2 A$ expression was associated with CD8+ T cells. The expression of CDKN3, CEP55, AURKA and UBE2C was related to the infiltration of macrophages. The expression of $A U R K A$ and BIRC5 was related to the infiltration of dendritic cells. Thus, the expression of any hub gene was associated with the infiltration of at least two immune cells.

\section{Validation of the hub genes via qRT-PCR}

The qRT-PCR was performed to further validate the expression of hub genes. As shown in Figure 10, the relative expression levels of $C D K N 3, M K I 67, C E P 55, S P A G 5$, AURKA, TOP2A were consistent with the results of bioinformatics analysis $(P<0.05)$, while the expression levels of $U B E 2 C$, CHEK1 and BIRC5 in tumor samples were not significantly different from adjacent normal samples.

\section{Discussion}

Lung cancer is still a common cause of health issues worldwide (Ma et al.,2020). So far, many lung cancer studies based on gene arrays have been conducted by different researchers, forming a series of gene expression datasets. By integrating multiple datasets, key genes involved in the progression and prognosis of lung cancer can be fully identified (Jin et al.,2020; Jin et al.,2020; Wu et al.,2020). We analyzed GEO datasets from the Chinese lung population and used bioinformatics to discover possible biomarkers of lung cancer. 
In this study, we analyzed 6 GEO datasets including GSE136043, GSE130779, GSE118370, GSE85841, GSE85716, and GSE89039, and a lot of 499 overlapping DEGs (160 upregulated and 339 downregulated genes) were identified among the datasets. The GO enrichment analysis indicated that the overlapping DEGs were mainly associated with angiogenesis, extracellular region, and heparin binding. Angiogenesis is a complex process, which plays a key role in maintaining tumor microenvironment, tumor growth, invasion and metastasis (Yu and Tian,2020). With a large number of studies on individual proteins, heparin-binding proteins (HBPs) have been proven to be important signaling molecules in the cell microenvironment affect the basic biological processes of development, homeostasis, and diseases (Nunes et al.,2019). The KEGG enrichment analysis indicated that the overlapping DEGs were mainly enriched in pathways in cancer, focal adhesion, and protein digestion and absorption. We extracted 9 hub genes (CDKN3, MKI67, CEP55, SPAG5, AURKA, TOP2A, UBE2C, CHEK1 and BIRC5) through PPI and module analysis. All of these genes were up-regulated in LUAD based on GEPIA database. And up-regulation of nine seven hub genes were associated with a poor prognosis of LUAD. Based on the HPA database, we found that the protein expression levels of most hub genes were higher in LUAD. Based on the ROC analysis, our results showed that all 9 hub genes (CDKN3, MKI67, CEP55, SPAG5, AURKA, TOP2A, UBE2C, CHEK1 and BIRC5) have good diagnostic efficiency in LUAD. The qRT-PCR analysis showed that the relative expression levels of CDKN3, MKI67, CEP55, SPAG5, AURKA, and TOP2A were consistent with the results of bioinformatics analysis.

The cyclin-dependent kinase inhibitor $3(C D K N 3)$ gene encodes a bi-specific protein, tyrosine phosphatase, that plays a key role in cell cycle and proliferation (Yu et al.,2020). CDKN3 overexpression is prognostic of poor overall survival in lung adenocarcinoma (Fan et al.,2015). Ki-67 is expressed in the active phases of the cell cycle, including G1, G2 and S, and has been used as an independent biomarker to predict prognosis in patients with lung cancer (Zheng et al.,2021). The centromeric protein CEP55, encoded by the CEP55, is widely expressed in different types of tissues, especially in proliferating tissues (Eloubeidi et al.,2002). CEP55 can be used as a diagnostic marker for LUAD and LUSC, but only as an independent prognostic factor for LUAD rather than LUSC (Fu et al.,2020). Sperm-associated antigen 5 (SPAG5, also known as asstrin) is involved in mitotic spindle formation and chromosome segregation, and has carcinogenic effects 
271 for the control of mitotic progression, centrosomal maturation/separation, and mitotic spindle function (Miralaei 272 et al.,2021). Studies have found that $A U R K A$ mRNA expression is an independent predictor of poor prognosis 273 in patients with NSCLC (Al-Khafaji et al.,2017). TOP2A, a cycle-dependent protein, is involved in a variety of 274 cell biological processes, such as DNA replication, chromatin condensation, chromosome separation, and 275 chromosome structure maintenance (Chen et al.,2015). TOP2A may be a prognostic biomarker and potential 276 therapeutic target for patients with LUAD (Du et al.,2020). We found that the expression levels of UBE2C,

$277 C H E K 1$, and BIRC5 in tumor samples were not significantly different from adjacent normal samples, which may 278 be due to the small sample size.

279 Analyzing GEO datasets of different populations may find different hub genes. A GEO data analysis of the

280

281

282

283

284

285 American LUAD population found 6 hub genes (VIPR1, FCN3, CA4, CRTAC1, CYP4B1, and NEDD9) related to prognosis (Jiawei et al.,2020). Another study on LUAD populations in Japan and USA found 8 hub genes (GPX3, TCN1, ASPM, PCP4, CAV2, S100P, COL1A1, and SPOK2) (Tu et al.,2021). These genes are different from those found in our study.

There are some limitations in our research, such as small sample size, lack of experimental validation in vivo and vitro, and no consideration of clinical information. More clinical samples and molecular experiments are needed in the future to conform the function of hub genes in lung cancer.

\section{Conclusions}

In conclusion, we filtrated a total of 499 overlapping DEGs from six GEO datasets and further validated 6 hub genes (CDKN3, MKI67, CEP55, SPAG5, AURKA, and TOP2A). The 6 hub genes were likely associated with the prognosis of lung patients in Chinese population. The functional pathways identified in the study may contribute to understand the molecular mechanisms of lung cancer. Our findings may provide new therapeutic targets for lung cancer patients.

\section{Conflict of interest}

The authors declare that they have no conflict of interests.

\section{Acknowledgments}


298

299

300

301

302

303

304

305

306

307

308

309

310

311

312

313

314

315

316

317

318

319

320

321

322

323

324

325

326

327

328

329

330

331

332

333

334

335

We thank MyGene Diagnostics Co., Ltd. for the technological assistance.

Funding

This study was supported by Pearl River S\&T Nova Program of Guangzhou (grant no. 201906010020)

\section{References}

Al-Khafaji ASK, Marcus MW, Davies MPA, Risk JM, Shaw RJ, Field JK, Liloglou T. 2017. AURKA mRNA expression is an independent predictor of poor prognosis in patients with non-small cell lung cancer. Oncol Lett 13(6): 4463-4468 DOI 10.3892/ol.2017.6012.

Chen T, Sun Y, Ji P, Kopetz S, Zhang W. 2015. Topoisomerase II $\alpha$ in chromosome instability and personalized cancer therapy. Oncogene 34(31): 4019-4031 DOI 10.1038/onc.2014.332.

Dai H, Guo L, Lin M, Cheng Z, Li J, Tang J, Huan X, Huang Y, Xu K. 2020. Comprehensive analysis and identification of key genes and signaling pathways in the occurrence and metastasis of cutaneous melanoma. PeerJ $\mathbf{8}$ : e10265 DOI 10.7717/peerj.10265.

Du X, Xue Z, Lv J, Wang H. 2020. Expression of the Topoisomerase II Alpha (TOP2A) Gene in Lung Adenocarcinoma Cells and the Association with Patient Outcomes. Med Sci Monit 26: e929120 DOI 10.12659/msm.929120.

Eloubeidi MA, Desmond R, Arguedas MR, Reed CE, Wilcox CM. 2002. Prognostic factors for the survival of patients with esophageal carcinoma in the U.S.: the importance of tumor length and lymph node status. Cancer 95(7): 1434-1443 DOI 10.1002/cncr.10868.

Fan C, Chen L, Huang Q, Shen T, Welsh EA, Teer JK, Cai J, Cress WD, Wu J. 2015. Overexpression of major CDKN3 transcripts is associated with poor survival in lung adenocarcinoma. British journal of cancer 113(12): 17351743 DOI 10.1038/bjc.2015.378.

Fu L, Wang H, Wei D, Wang B, Zhang C, Zhu T, Ma Z, Li Z, Wu Y, Yu G. 2020. The value of CEP55 gene as a diagnostic biomarker and independent prognostic factor in LUAD and LUSC. PLoS One 15(5): e0233283 DOI 10.1371/journal.pone.0233283.

Gu W, Sun Y, Zheng X, Ma J, Hu X-Y, Gao T, Hu M-J. 2018. Identification of Gastric Cancer-Related Circular RNA through Microarray Analysis and Bioinformatics Analysis. BioMed research international 2018: 2381680 DOI $10.1155 / 2018 / 2381680$.

Hirsch FR, Suda K, Wiens J, Bunn PA. 2016. New and emerging targeted treatments in advanced non-small-cell lung cancer. Lancet (London, England) 388(10048): 1012-1024 DOI 10.1016/S0140-6736(16)31473-8.

Huang DW, Sherman BT, Lempicki RA. 2009. Systematic and integrative analysis of large gene lists using DAVID bioinformatics resources. Nature protocols 4(1): 44-57 DOI 10.1038/nprot.2008.211.

Huang R, Li A. 2020. SPAG5 is associated with unfavorable prognosis in patients with lung adenocarcinoma and promotes proliferation, motility and autophagy in A549 cells. Exp Ther Med 20(5): 77 DOI 10.3892/etm.2020.9205.

Jia Y, Liu Y, Han Z, Tian R. 2021. Identification of potential gene signatures associated with osteosarcoma by integrated bioinformatics analysis. PeerJ 9: e11496 DOI 10.7717/peerj.11496.

Jiao Z, Yu A, He X, Xuan Y, Zhang H, Wang G, Shi M, Wang T. 2020. Bioinformatics analysis to determine the 
prognostic value and prospective pathway signaling of miR-126 in non-small cell lung cancer. Annals of translational medicine 8(24): 1639 DOI 10.21037/atm-20-7520.

Jiawei Z, Min M, Yingru X, Xin Z, Danting L, Yafeng L, Jun X, Wangfa H, Lijun Z, Jing W, Dong H. 2020. Identification of Key Genes in Lung Adenocarcinoma and Establishment of Prognostic Mode. Frontiers in molecular biosciences 7: 561456 DOI 10.3389/fmolb.2020.561456.

Jin D, Song Y, Chen Y, Zhang P. 2020. Identification of Three IncRNAs as Potential Predictive Biomarkers of Lung Adenocarcinoma. BioMed research international 2020: 7573689 DOI 10.1155/2020/7573689.

Jin X, Guan Y, Zhang Z, Wang H. 2020. Microarray data analysis on gene and miRNA expression to identify biomarkers in non-small cell lung cancer. BMC cancer 20(1): 329 DOI 10.1186/s12885-020-06829-x.

Kolde R, Laur S, Adler P, Vilo J. 2012. Robust rank aggregation for gene list integration and meta-analysis. Bioinformatics (Oxford, England) 28(4): 573-580 DOI 10.1093/bioinformatics/btr709.

Li M, Wang X, Liu J, Mao X, Li D, Wang Z, Tang Y, Wu S. 2020. Identification of Core Prognosis-Related Candidate Genes in Chinese Gastric Cancer Population Based on Integrated Bioinformatics. BioMed research international 2020: 8859826 DOI 10.1155/2020/8859826.

Li Z, Qi F, Li F. 2020. Establishment of a Gene Signature to Predict Prognosis for Patients with Lung Adenocarcinoma. International journal of molecular sciences 21(22) DOI 10.3390/ijms21228479.

Liu K, Fu Q, Liu Y, Wang C. 2019. An integrative bioinformatics analysis of microarray data for identifying hub genes as diagnostic biomarkers of preeclampsia. Bioscience reports 39(9) DOI 10.1042/BSR20190187.

Liu S, Liu X, Wu J, Zhou W, Ni M, Meng Z, Jia S, Zhang J, Guo S, Lu S, Li Y. 2020. Identification of candidate biomarkers correlated with the pathogenesis and prognosis of breast cancer via integrated bioinformatics analysis. Medicine 99(49): e23153 DOI 10.1097/MD.0000000000023153.

Ma X, Ren H, Peng R, Li Y, Ming L. 2020. Identification of key genes associated with progression and prognosis for lung squamous cell carcinoma. PeerJ 8: e9086 DOI 10.7717/peerj.9086.

Ma Z, Xu J, Ru L, Zhu W. 2021. Identification of pivotal genes associated with the prognosis of gastric carcinoma through integrated analysis. Bioscience reports 41(4) DOI 10.1042/bsr20203676.

Miralaei N, Majd A, Ghaedi K, Peymani M, Safaei M. 2021. Integrated pan-cancer of AURKA expression and drug sensitivity analysis reveals increased expression of AURKA is responsible for drug resistance. Cancer Med DOI 10.1002/cam4.4161.

Mou Y, Wu J, Zhang Y, Abdihamid O, Duan C, Li B. 2021. Low expression of ferritinophagy-related NCOA4 gene in relation to unfavorable outcome and defective immune cells infiltration in clear cell renal carcinoma. $B M C$ cancer 21(1): 18 DOI 10.1186/s12885-020-07726-z.

Nunes QM, Su D, Brownridge PJ, Simpson DM, Sun C, Li Y, Bui TP, Zhang X, Huang W, Rigden DJ, Beynon RJ, Sutton R, Fernig DG. 2019. The heparin-binding proteome in normal pancreas and murine experimental acute pancreatitis. PLoS One 14(6): e0217633 DOI 10.1371/journal.pone.0217633.

Parida S, Siddharth S, Sharma D. 2021. Role of Omentin in Obesity Paradox in Lung Cancer. Cancers 13(2) DOI 10.3390/cancers 13020275 .

Siegel RL, Miller KD, Jemal A. 2019. Cancer statistics, 2019. CA: a cancer journal for clinicians 69(1) DOI 10.3322/caac.21551.

Song Y, Tang W, Li H. 2021. Identification of KIF4A and its effect on the progression of lung adenocarcinoma based on the bioinformatics analysis. Bioscience reports 41(1) DOI 10.1042/BSR20203973.

Sun D, Li H, Cao M, He S, Lei L, Peng J, Chen W. 2020. Cancer burden in China: trends, risk factors and prevention. 
377 Cancer biology \& medicine 17(4): 879-895 DOI 10.20892/j.issn.2095-3941.2020.0387.

378 Szklarczyk D, Gable AL, Lyon D, Junge A, Wyder S, Huerta-Cepas J, Simonovic M, Doncheva NT, Morris JH,

379 Bork P, Jensen LJ, Mering Cv. 2019. STRING v11: protein-protein association networks with increased coverage,

380 supporting functional discovery in genome-wide experimental datasets. Nucleic acids research 47(D1): D607-D613

381 DOI 10.1093/nar/gky1131.

382 Tang S, Jing H, Huang Z, Huang T, Lin S, Liao M, Zhou J. 2020. Identification of key candidate genes in 383 neuropathic pain by integrated bioinformatic analysis. Journal of cellular biochemistry 121(2): 1635-1648 DOI $38410.1002 /$ jcb.29398.

385 Travis WD. 2011. Pathology of lung cancer. Clinics in chest medicine 32(4): 669-692 DOI 386 10.1016/j.ccm.2011.08.005.

387 Tu Z, He X, Zeng L, Meng D, Zhuang R, Zhao J, Dai W. 2021. Exploration of Prognostic Biomarkers for Lung 388 Adenocarcinoma Through Bioinformatics Analysis. Front Genet 12: 647521 DOI 10.3389/fgene.2021.647521.

389 Wang X, Chen X, Liu H. 2020. Expression and Bioinformatics-Based Functional Analysis of UAP1 in Lung 390 Adenocarcinoma. Cancer management and research 12: 12111-12121 DOI 10.2147/CMAR.S282238.

391 Wu Z, Wang Y-M, Dai Y, Chen L-A. 2020. POLE2 Serves as a Prognostic Biomarker and Is Associated with 392 Immune Infiltration in Squamous Cell Lung Cancer. Medical science monitor : international medical journal of 393 experimental and clinical research 26: e921430 DOI 10.12659/MSM.921430.

394 Yang R, Zhou Y, Du C, Wu Y. 2020. Bioinformatics analysis of differentially expressed genes in tumor and 395 paracancerous tissues of patients with lung adenocarcinoma. Journal of thoracic disease 12(12): 7355-7364 DOI $39610.21037 /$ jtd-20-3453.

397 Yang X, Tian M, Zhang W, Chai T, Shen Z, Kang M, Lin J. 2021. Identification of potential core genes in 398 esophageal carcinoma using bioinformatics analysis. Medicine 100(27): e26428 DOI 399 10.1097/MD.0000000000026428.

400 Yu H, Yao J, Du M, Ye J, He X, Yin L. 2020. CDKN3 promotes cell proliferation, invasion and migration by 401 activating the AKT signaling pathway in esophageal squamous cell carcinoma. Oncol Lett 19(1): 542-548 DOI $402 \quad 10.3892 / \mathrm{ol} .2019 .11077$.

403 Yu Y, Tian X. 2020. Analysis of genes associated with prognosis of lung adenocarcinoma based on GEO and TCGA 404 databases. Medicine 99(19): e20183 DOI 10.1097/MD.0000000000020183.

405 Zheng Y, Huang W, Zhang X, Lu C, Fu C, Li S, Lin G. 2021. A Noninvasive Assessment of Tumor Proliferation 406 in Lung cancer Patients using Intravoxel Incoherent Motion Magnetic Resonance Imaging. J Cancer 12(1): 190-197 407 DOI 10.7150/jca.48589.

408 Zhu Y, McKeon TP, Tam V, Vachani A, Penning TM, Hwang W-T. 2020. Geographic Differences in Lung Cancer 409 Incidence: A Study of a Major Metropolitan Area within Southeastern Pennsylvania. International journal of 410 environmental research and public health 17(24) DOI 10.3390/ijerph17249498.

414 Figure 1 The volcano plots of DEGs in 6 datasets. The DEGs in (a) GSE136043 (B) GSE130779, (C) 415 GSE1 18370, and (D) GSE85841, (E) GSE85716 and (F) GSE89039 datasets. The red dots represent upregulated 
416

417

418

419

420

421

422

423

424

425

426

427

428

429

430

431

432

433

436

437

genes according to an adjusted $P<0.05$ and $\mid \log$ fold change $\mid>1$; the green dots represent downregulated genes according to an adjusted $P<0.05$ and $\mid \log$ fold change $\mid>1$; the black dots represent genes with no significant difference in expression. DEG, differentially expressed genes.

Figure 2 The top 20 up- and downregulated genes in integrated datasets. The abscissa represents the GEO datasets, and the ordinate represents the gene name. The red represents $\log \mathrm{FC}>0$; the pink represents $\log \mathrm{FC}$ is slightly less than 0 ; the green represents $\log \mathrm{FC}<0$.

Figure 3 The results of enrichment analysis. (A) The results of GO annotation analysis. (B) The KEGG pathway enrichment analysis of DEGs. GO, Gene ontology; KEGG, Kyoto Encyclopedia of Genes and Genomes; DEGs, differentially expressed genes.

Figure 4 PPI network construction and module analysis. (A) The PPI network of DEGs. The red circles represents the upregulated DEGs and blue circles represents the downregulated DEGs. (B) Module 1 from the PPI network. (C) Module 2 from the PPI network. (3) Module 3 from the PPI network. DEGs, differentially expressed genes; PPI, protein-protein interaction.

Figure 5 Validation of the mRNA expression of (A-I) CDKN3, MKI67, CEP55, SPAG5, AURKA, TOP2A, UBE2C, CHEK1, and BIRC5 in LUAD tissues and normal tissues using GEPIA database. LUAD, lung adenocarcinoma.

Figure 6 Prognostic value of (A-I) CDKN3, MKI67, CEP55, SPAG5 (DEEPEST), AURKA, TOP2A, UBE2C, CHEK1, and BIRC5 in LUAD patients. The prognostic information of the 9 hub genes in patients with LUAD was from Kaplan-Meier plotter database. LUAD, lung adenocarcinoma.

Figure 7 Immunohistochemistry images of hub genes in LUAD tissues and normal lung tissues derived 
443 from the HPA database. The protein images of (A-G) MKI67, CEP55, SPAG5, AURKA, TOP2A, UBE2C and 444 BIRC5 in HPA database. LUAD, lung adenocarcinoma; HPA, Human Protein Atlas.

445

Figure 8 ROC curves of hub genes in TCGA database. The ROC curves of (A-I) CDKN3, MKI67, CEP55, 447 SPAG5, AURKA, TOP2A, UBE2C, CHEK1, and BIRC5. ROC, receiver operating characteristic.

448

449

450

451

452

453

454

455

456

457

458

459

460 461 462 463

464

\section{0}

Figure 9 Correlation between the expression of (A-I) CDKN3, MKI67, CEP55, SPAG5, AURKA, TOP2A, UBE2C, CHEK1, BIRC5 and immune cell populations (B Cell, CD8+ T Cell, CD4+ T Cell, macrophage, neutrophil, and dendritic cell) in LUAD. $P<0.05$ was considered statistically significant.

Figure 10. RT-PCR validation of the hub genes between lung cancer tissues and normal controls $(* P<$ $0.05, * * P<0.01)$.

Figure S1 Normalization of gene expression matrix. (A) Normalization of the GSE136043 dataset. (B) Normalization of the GSE130779 dataset. (C) Normalization of the GSE118370 dataset. (D) Normalization of the GSE85841 dataset. (E) Normalization of the GSE85716 dataset. (F) Normalization of the GSE89039 dataset. Blue represents data before normalization, and red represents data after normalization. 


\section{Figure 1}

The volcano plots of DEGs in 6 datasets.

The DEGs in (a) GSE136043 (B) GSE130779, (C) GSE118370, and (D) GSE85841, (E) GSE85716 and (F) GSE89039 datasets. The red dots represent upregulated genes according to an adjusted $\mathrm{P}<0.05$ and $\mid \log$ fold change $>1$; the green dots represent downregulated genes according to an adjusted $\mathrm{P}<0.05$ and $\mid \log$ fold change $\mid>1$; the black dots represent genes with no significant difference in expression. DEG, differentially expressed genes. 

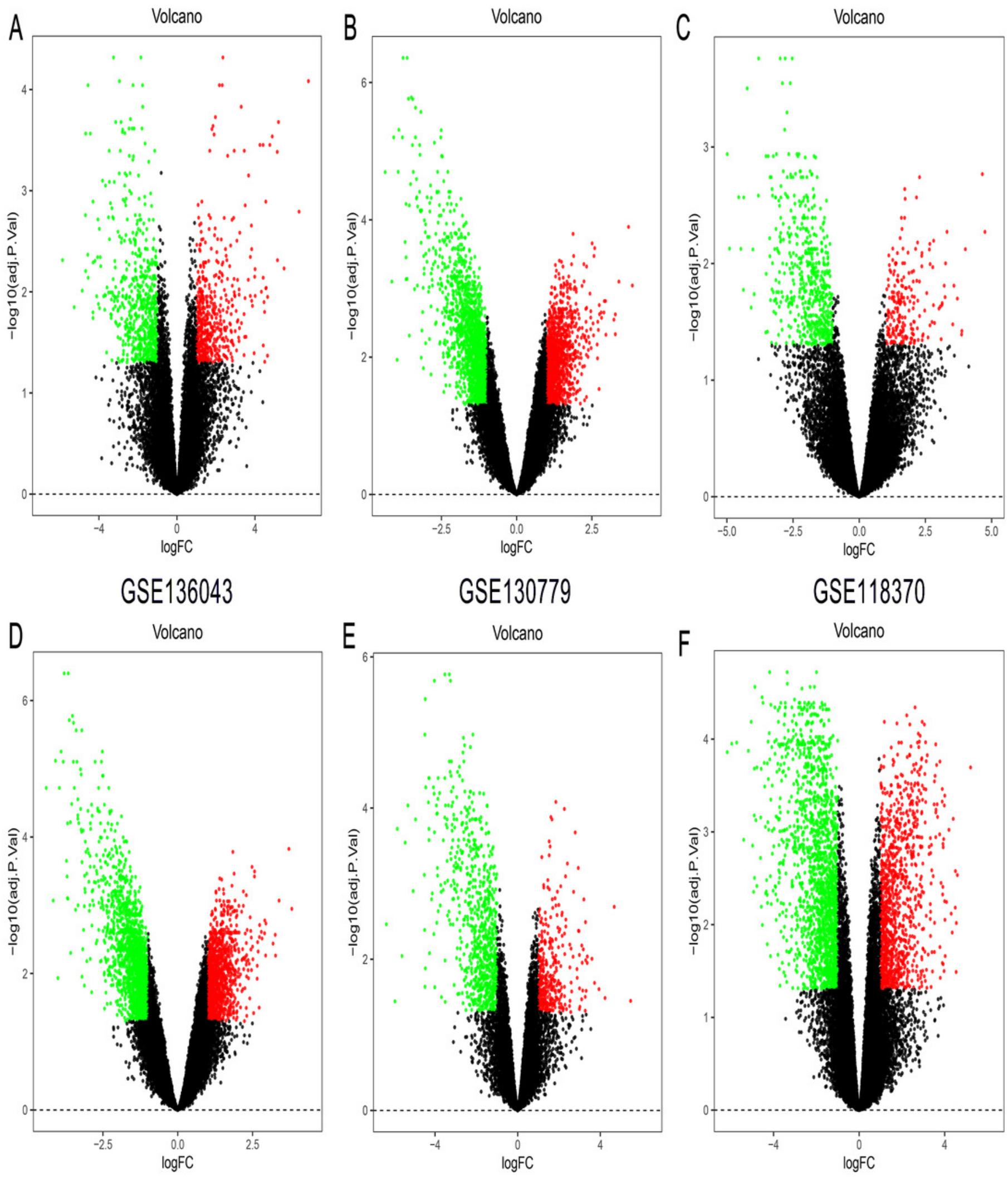

GSE85841

GSE85716

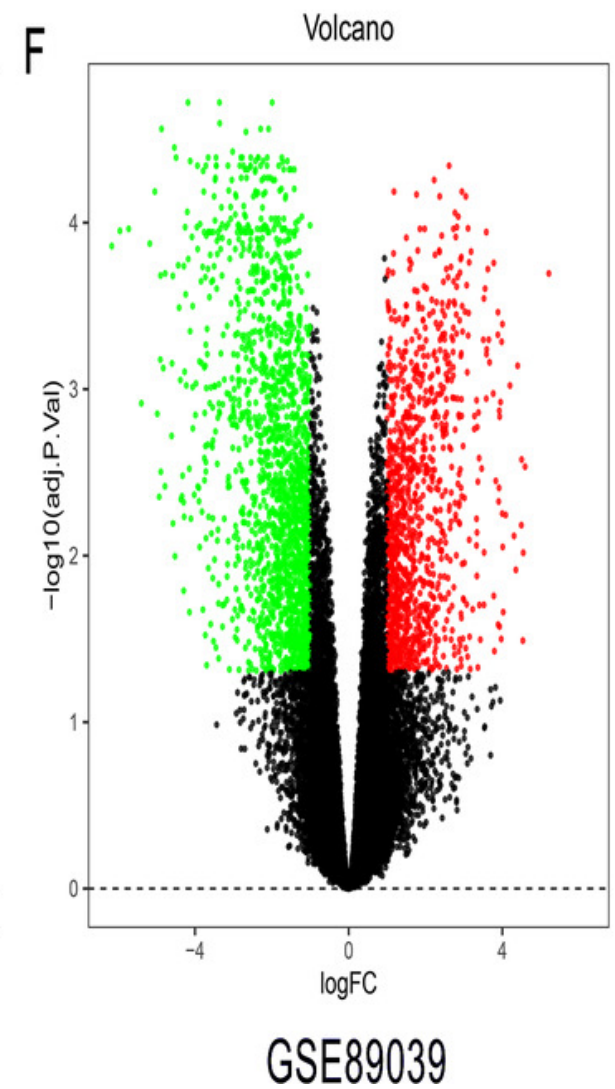


Figure 2

The top 20 up- and downregulated genes in integrated datasets.

The abscissa represents the GEO datasets, and the ordinate represents the gene name. The red represents $\log F C>0$; the pink represents $\log F C$ is slightly less than 0 ; the green represents $\log \mathrm{FC}<0$. 


\begin{tabular}{|c|c|c|c|c|c|c|}
\hline & 3.40 & 3.23 & 3.39 & 4.68 & 2.27 & SPP1 \\
\hline 2.54 & 2.47 & 2.55 & 2.44 & 1.86 & 2.87 & HABP2 \\
\hline & 1.90 & 2.19 & 1.89 & 2.57 & 2.86 & CEACAM5 \\
\hline 5.49 & 2.04 & 2.13 & 2.03 & 1.94 & 3.67 & COL10A1 \\
\hline 3.30 & 2.44 & 0.95 & 2.42 & 3.32 & 3.83 & PITX1 \\
\hline 1.95 & 3.72 & 1.98 & 3.72 & 1.80 & 3.92 & CST2 \\
\hline 3.04 & 2.42 & 2.21 & 2.40 & 2.12 & 0.00 & SERPIND1 \\
\hline 3.76 & 0.71 & 3.32 & 0.68 & 3.10 & 3.98 & TOX3 \\
\hline 1.95 & 1.91 & 1.54 & 1.89 & 2.33 & 2.30 & STK32A \\
\hline 4.58 & 1.80 & 2.61 & 1.76 & 0.46 & 3.23 & TMEM63C \\
\hline 2.99 & 2.30 & 2.12 & 2.26 & 1.75 & 1.34 & $\mathrm{CDH} 3$ \\
\hline-1.22 & 3.28 & 1.44 & 3.26 & 0.00 & 5.21 & GCNT3 \\
\hline 3.96 & 0.68 & 2.74 & 0.66 & 2.87 & 4.30 & CXCL13 \\
\hline 3.19 & 1.88 & 0.11 & 1.86 & 3.54 & 2.51 & EEF1A2 \\
\hline 3.17 & 2.68 & 1.97 & 2.66 & 2.34 & 0.60 & LRRC31 \\
\hline 1.99 & 3.28 & 2.65 & 3.27 & 3.10 & 1.10 & ABCC3 \\
\hline 2.75 & 2.70 & 0.21 & 2.67 & 4.23 & 2.44 & TUBB3 \\
\hline 2.54 & 2.75 & 1.93 & 2.74 & 1.82 & -0.16 & CXCL14 \\
\hline 2.08 & 1.77 & 2.35 & 1.76 & 0.00 & 3.58 & CST1 \\
\hline 3.16 & 2.32 & 0.00 & 2.31 & 5.47 & 0.94 & XAGE1A \\
\hline-4.03 & -3.60 & -3.37 & -3.62 & -5.60 & -4.57 & CLDN18 \\
\hline-3.82 & -2.38 & -3.03 & -2.39 & -4.49 & -4.41 & AGER \\
\hline-4.07 & -2.59 & -3.28 & -2.61 & -3.86 & -3.36 & RTKN2 \\
\hline-3.61 & -3.92 & -1.93 & -3.90 & -4.29 & -4.93 & ADAMTS8 \\
\hline-3.59 & -3.68 & 0.00 & -3.68 & -5.06 & -4.30 & FIGF \\
\hline-3.50 & -3.02 & -3.02 & -3.04 & -4.30 & -3.14 & GRIA1 \\
\hline-3.33 & -2.27 & -4.55 & -2.30 & -4.94 & -3.05 & TMEM100 \\
\hline-4.56 & -2.16 & -4.47 & -2.19 & -4.03 & -6.17 & SGCG \\
\hline-3.06 & -2.14 & -3.23 & -2.16 & -3.52 & -3.06 & LYVE1 \\
\hline-3.22 & -2.13 & -2.62 & -2.16 & -3.41 & -3.38 & STXBP6 \\
\hline-2.39 & -3.18 & -2.28 & -3.20 & -5.45 & -4.18 & FAM107A \\
\hline-2.36 & -2.93 & -2.33 & -2.95 & -4.47 & -3.14 & ADRB1 \\
\hline-2.33 & -3.67 & -2.34 & -3.69 & -3.93 & -4.18 & TCF21 \\
\hline-5.87 & -2.89 & 0.00 & -2.91 & -4.52 & -5.73 & ITLN2 \\
\hline-3.18 & -2.01 & -2.35 & -2.04 & -3.19 & -3.11 & CDO1 \\
\hline-2.20 & -3.19 & -2.50 & -3.20 & -4.03 & -2.90 & FMO2 \\
\hline-3.46 & -2.63 & -2.26 & -2.64 & -2.09 & -3.99 & RBP2 \\
\hline-2.14 & -2.16 & -2.48 & -2.18 & -3.85 & -3.48 & SOX7 \\
\hline-2.94 & -3.78 & -1.49 & -3.79 & -3.78 & -4.34 & $\mathrm{ADH} 1 \mathrm{~A}$ \\
\hline-3.15 & -2.14 & -1.95 & -2.18 & -4.17 & -3.65 & MYZAP \\
\hline 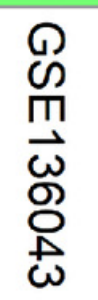 & 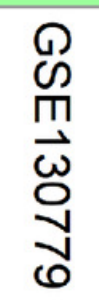 & 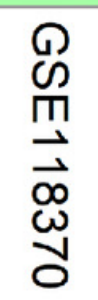 & 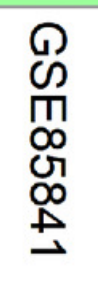 & 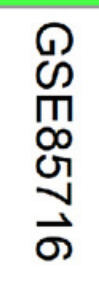 & 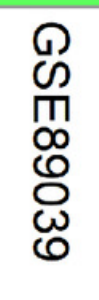 & \\
\hline
\end{tabular}


Figure 3

The results of enrichment analysis.

(A) The results of GO annotation analysis. (B) The KEGG pathway enrichment analysis of DEGs. GO, Gene ontology; KEGG, Kyoto Encyclopedia of Genes and Genomes; DEGs, differentially expressed genes. 
A

collagen binding

metalloendopeptidase activity

calcium ion binding

heparin binding

integral component of plasma membrane

E⿱ matrix

extracellular region negative regulation of endothelial cell proliferation response to drug -

cell adhesion -

collagen catabolic process

angiogenesis

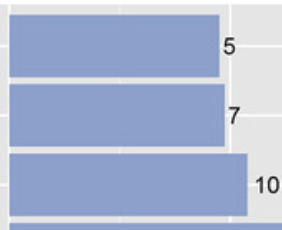

The Most enriched GO Terms

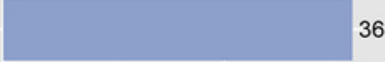

36

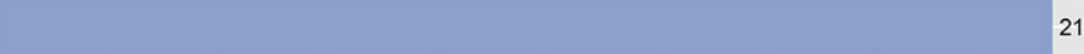

64
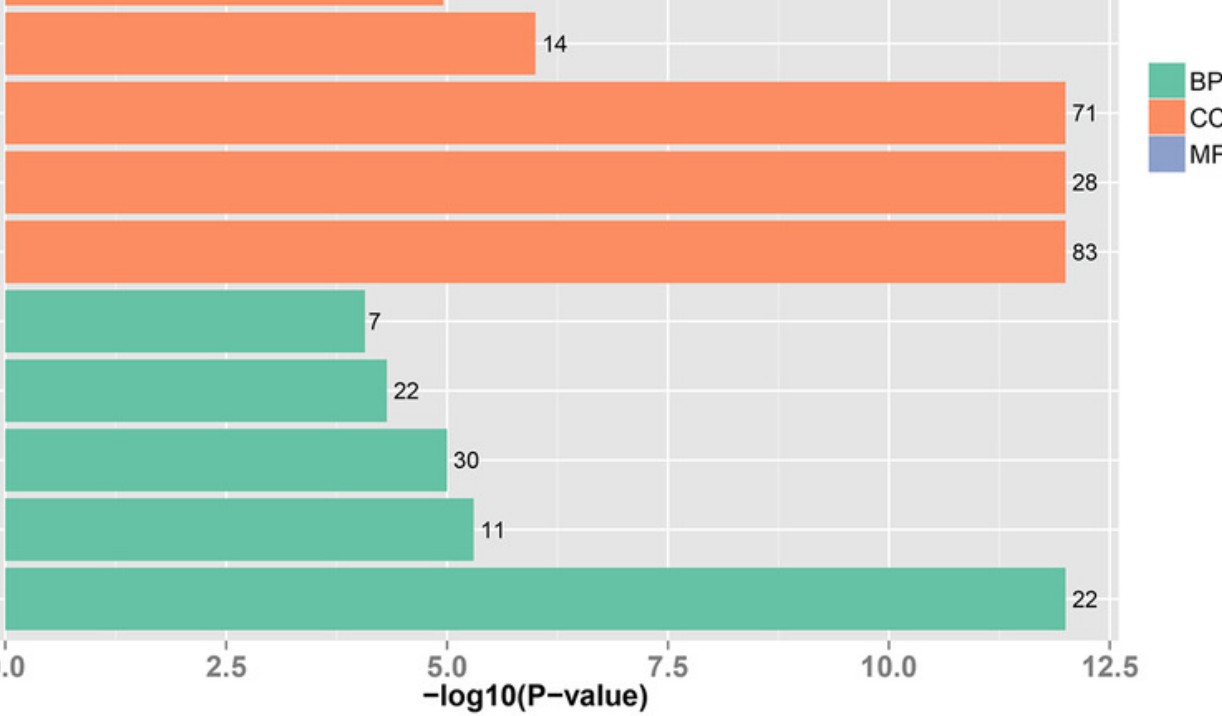

B

Tyrosine metabolism Transcriptional misregulation in cancer Salivary secretion -

Renin secretion

Protein digestion and absorption

PPAR signaling pathway

Pathways in cancer

Malaria -

Focal adhesion

ECM-receptor interaction

Drug metabolism - cytochrome P450 Complement and coagulation cascades -

cGMP-PKG signaling pathway

cAMP signaling pathway

Bile secretion

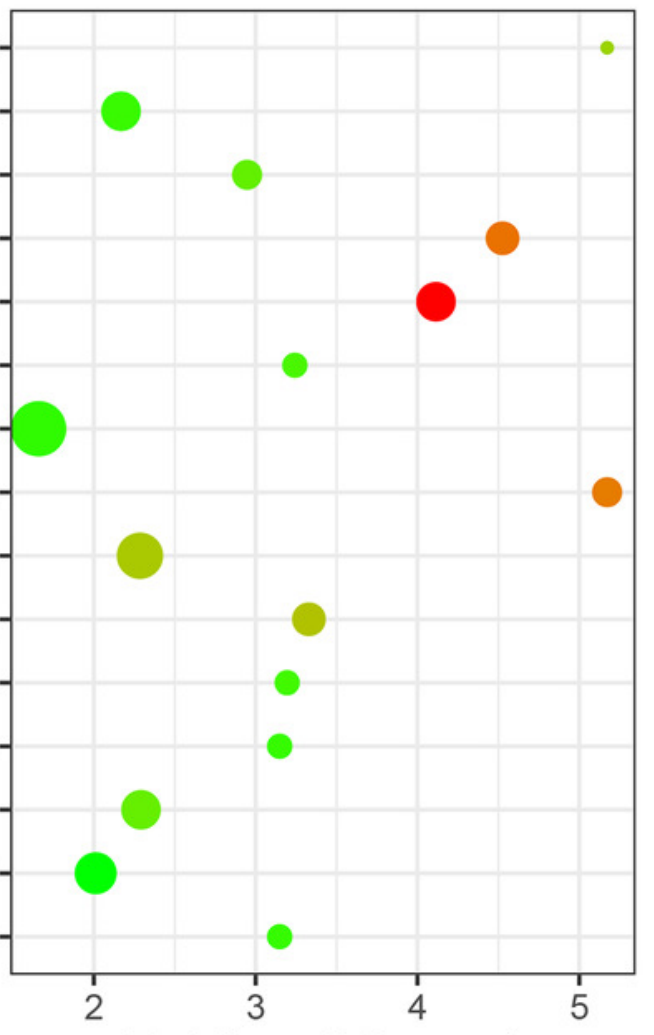

$-\log _{10}$ (P.value)

3.0

2.5

2.0

1.5

Gene number

- 5.0

- 7.5

10.0

12.5

Fold enrichment

15.0

17.5 
Figure 4

PPI network construction and module analysis.

(A) The PPI network of DEGs. The red circles represents the upregulated DEGs and blue circles represents the downregulated DEGs. (B) Module 1 from the PPI network. (C) Module 2 from the PPI network. (3) Module 3 from the PPI network. DEGs, differentially expressed genes; PPI, protein-protein interaction. 
A
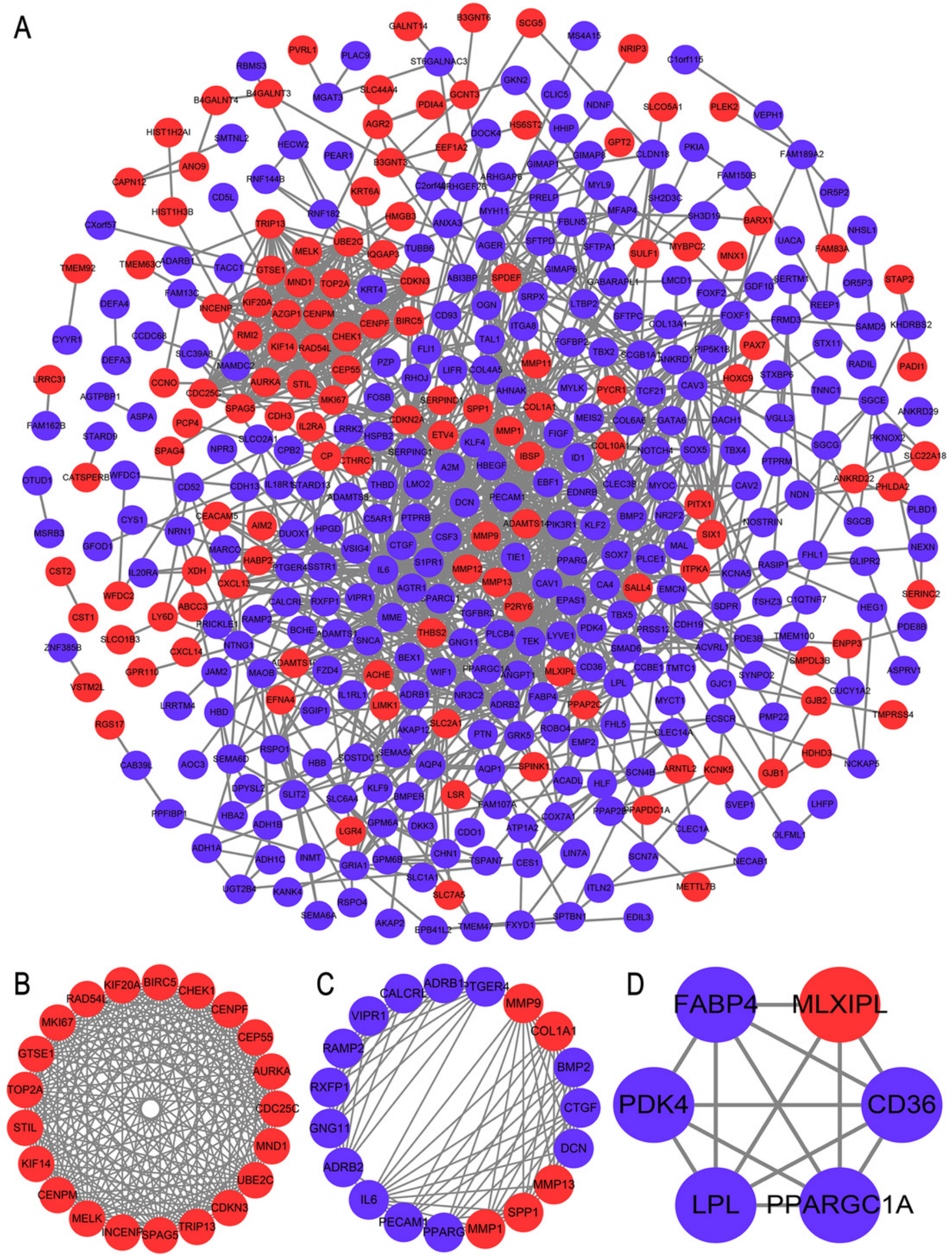

Peer) reviewing PDF | (2021:04:60758:2:0:NEW 26 Nov 2021) 
Figure 5

Validation of the mRNA expression of (A-I) CDKN3, MKI67, CEP55, SPAG5, AURKA, TOP2A, UBE2C, CHEK1, and BIRC5 in LUAD tissues and normal tissues using GEPIA database. LUAD, lung adenocarcinoma. 

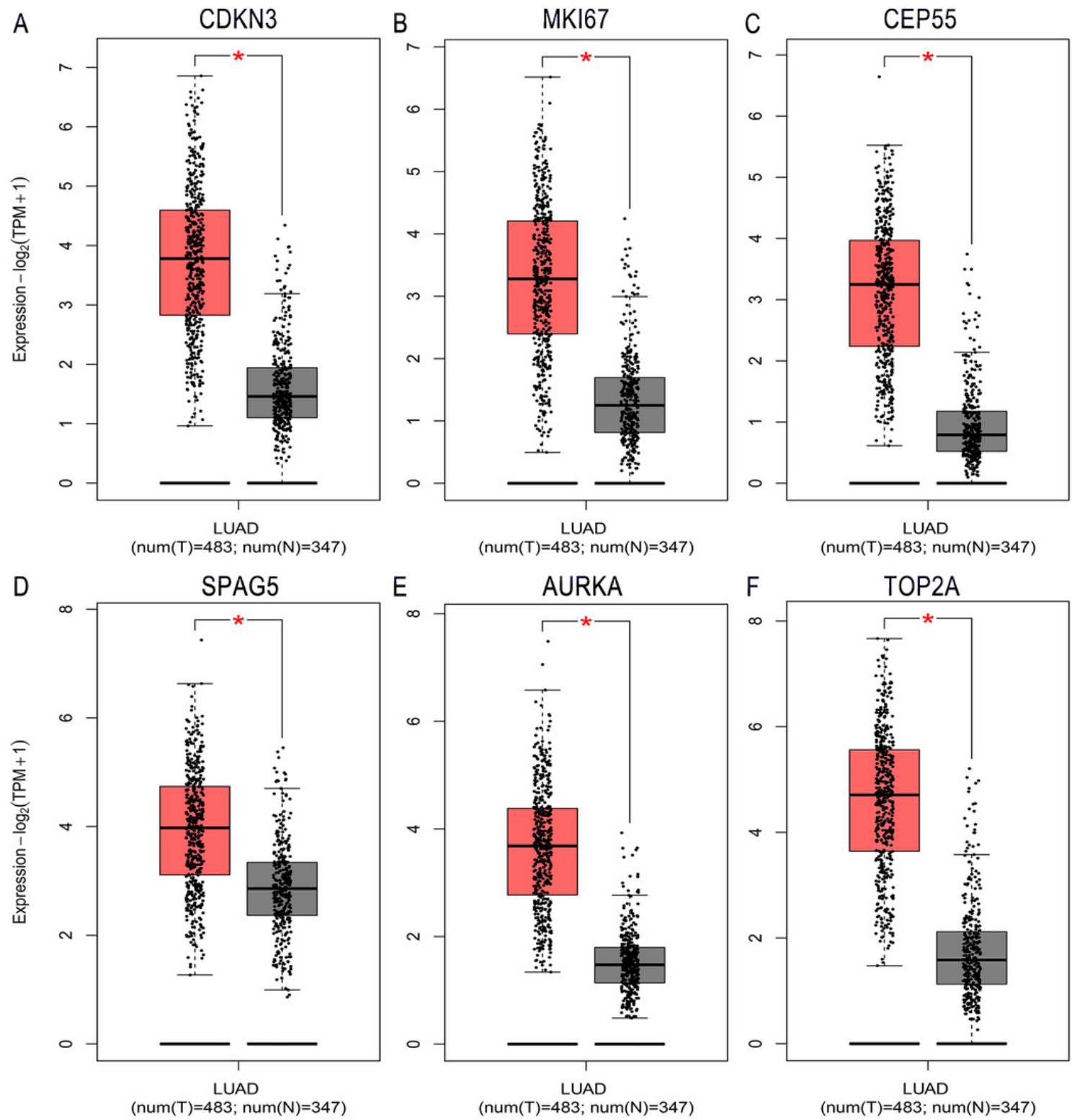

(num $(T)=483 ; \operatorname{num}(N)=347$ )
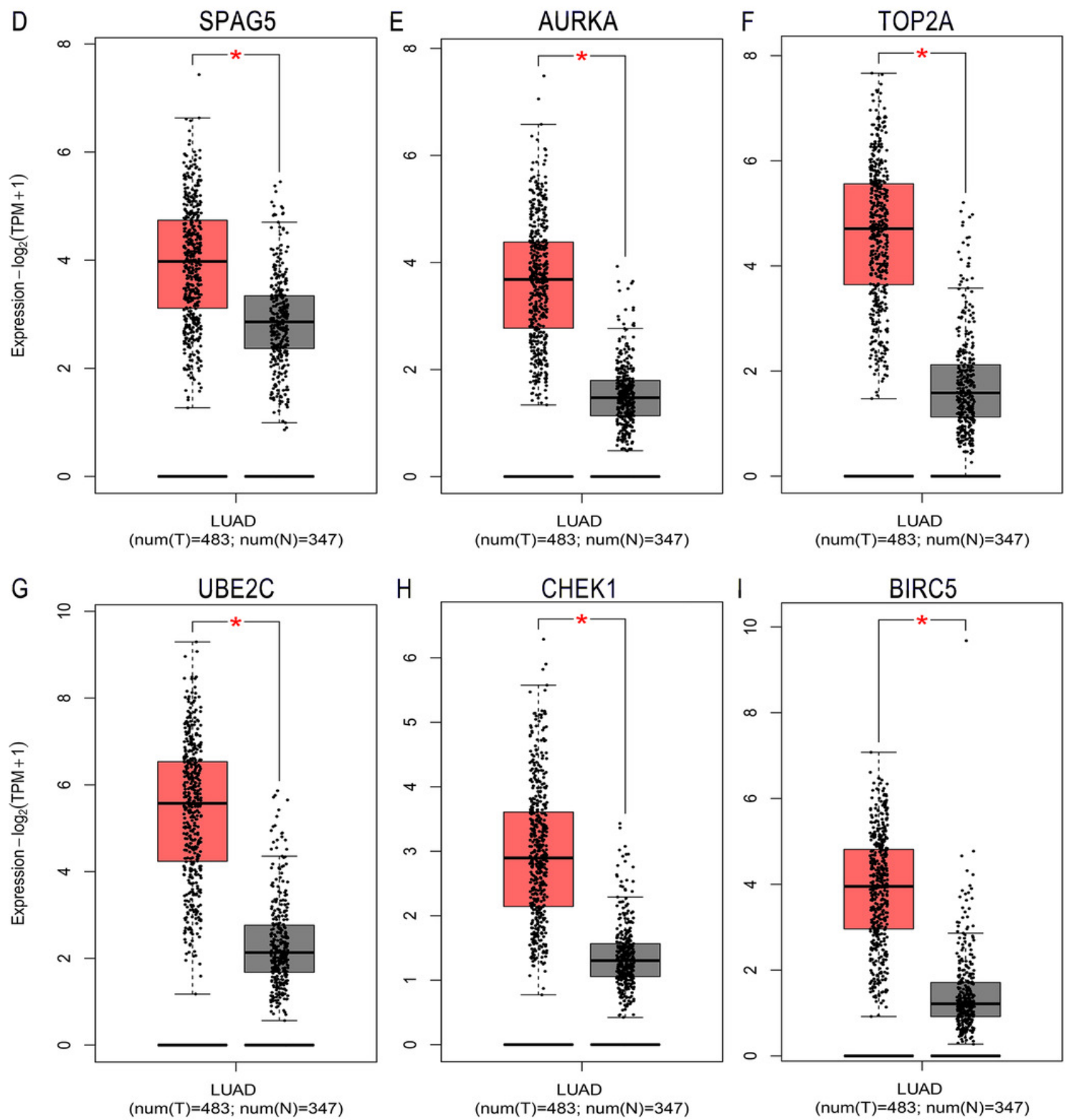

Peer] reviewing PDF | (2021:04:60758:2:0:NEW 26 Nov 2021) 
Figure 6

Prognostic value of (A-I) CDKN3, MKI67, CEP55, SPAG5 (DEEPEST), AURKA, TOP2A, UBE2C, CHEK1, and BIRC5 in LUAD patients.

The prognostic information of the 9 hub genes in patients with LUAD was from Kaplan-Meier plotter database. LUAD, lung adenocarcinoma. 

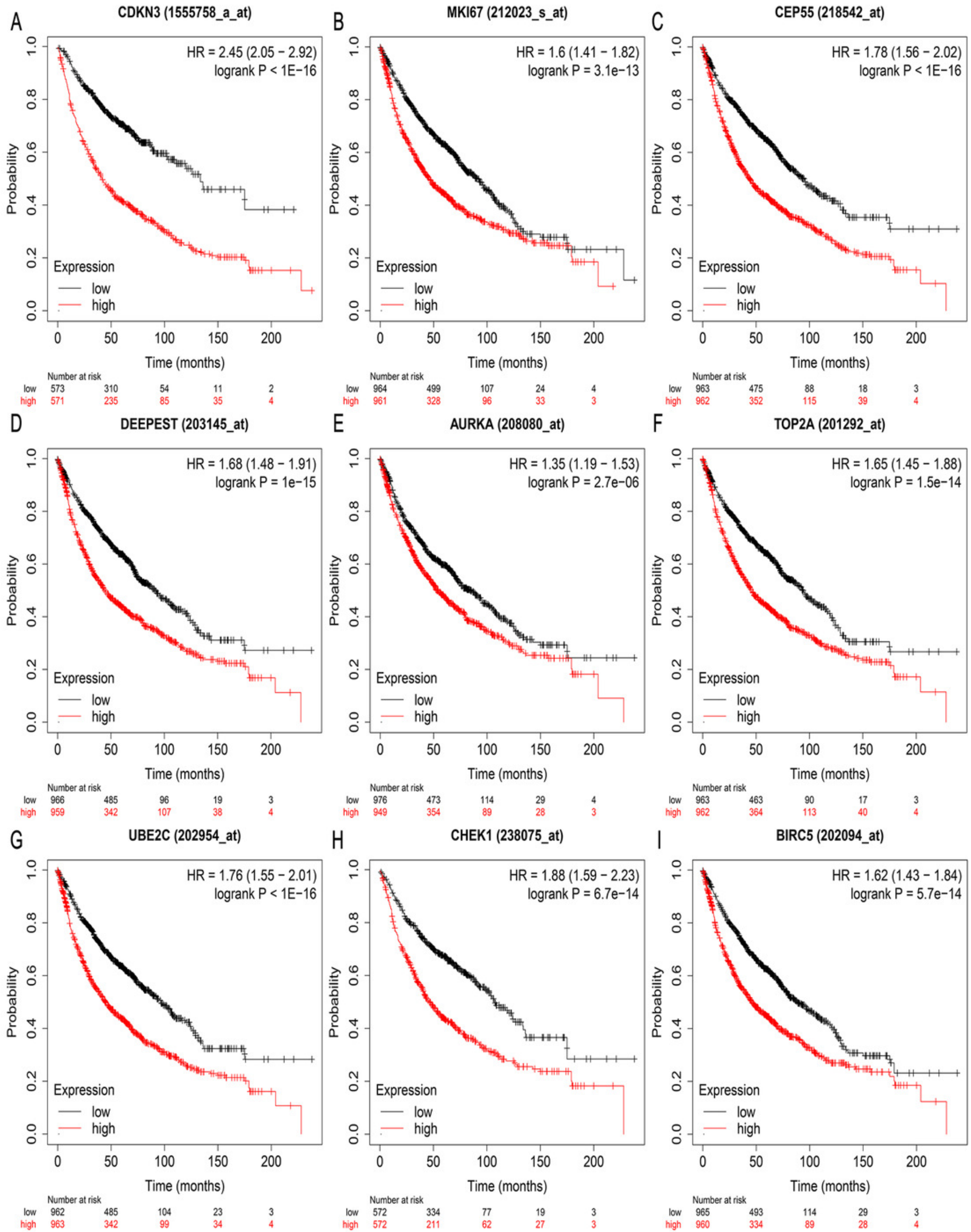


\section{Figure 7}

Immunohistochemistry images of hub genes in LUAD tissues and normal lung tissues derived from the HPA database.

The protein images of (A-G) MKI67, CEP55, SPAG5, AURKA, TOP2A, UBE2C and BIRC5 in HPA database. LUAD, lung adenocarcinoma; HPA, Human Protein Atlas. 

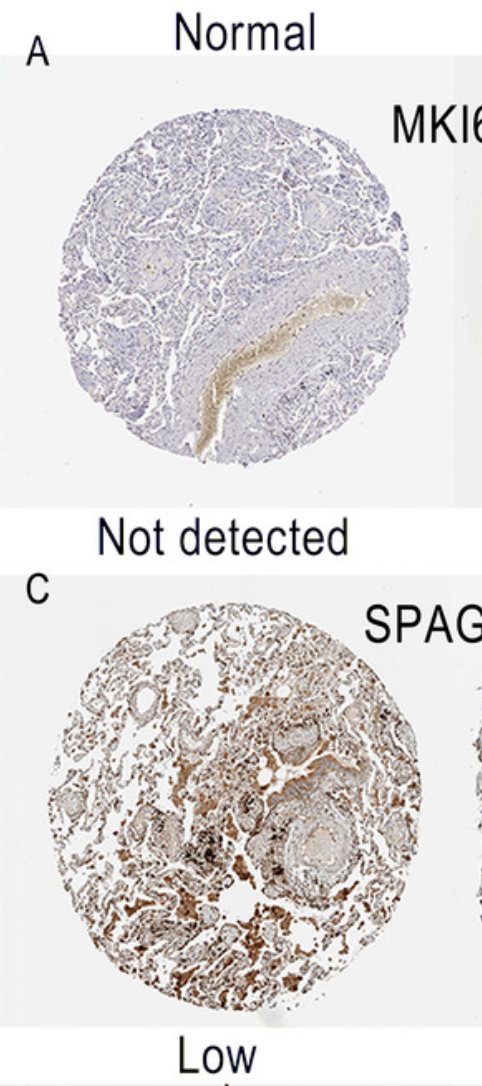

E

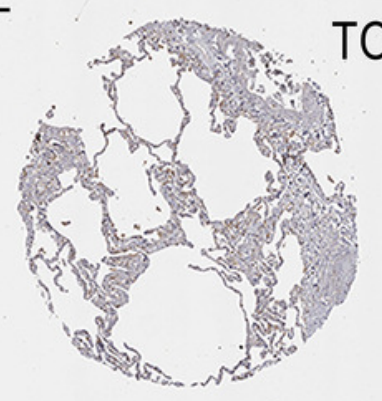

Low

LUAD

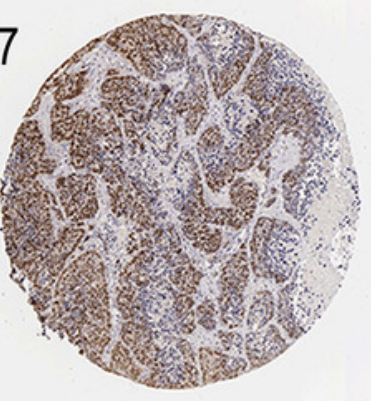

High

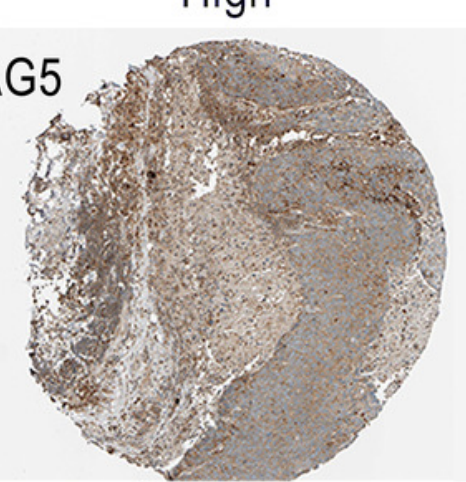

Medium

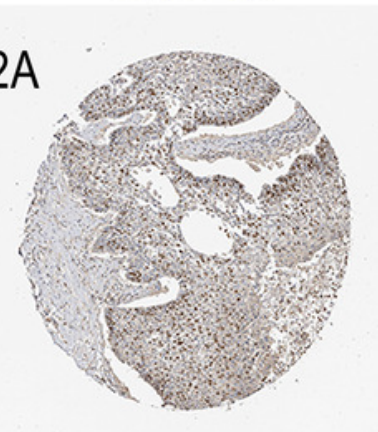

High

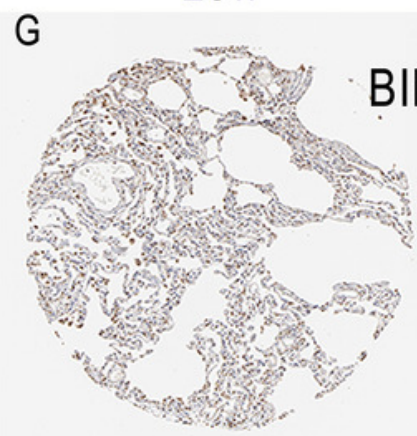

Not detected
B Normal LUAD

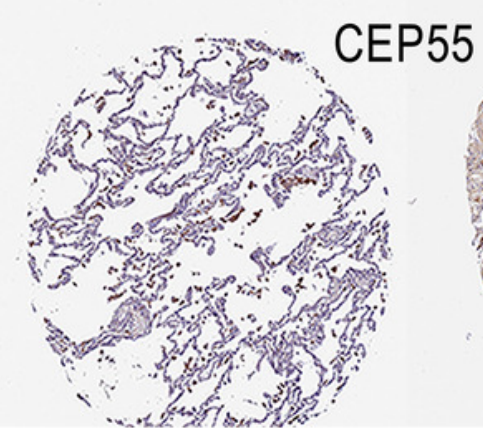

Not detected

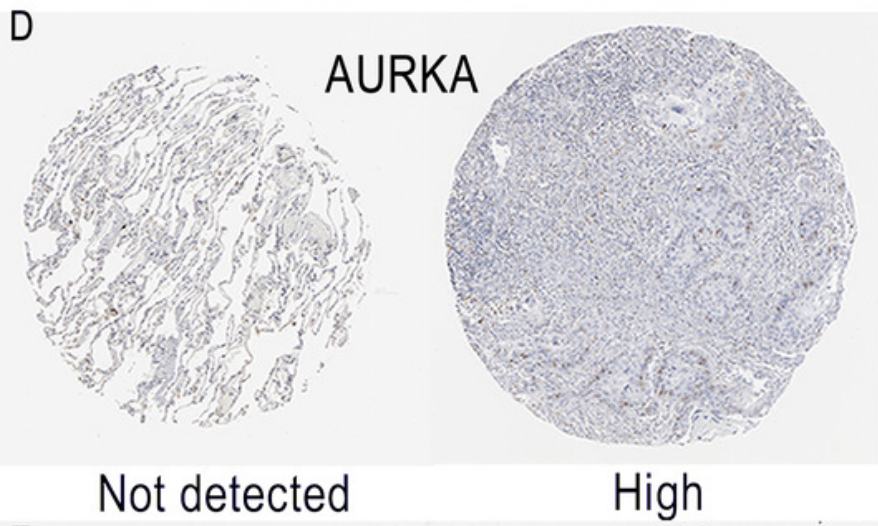

F

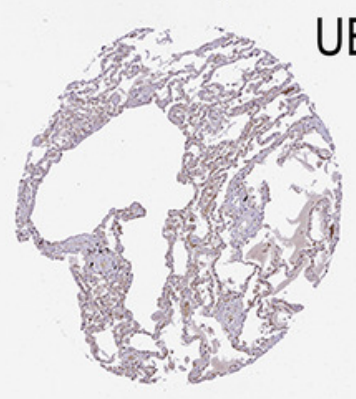

Low

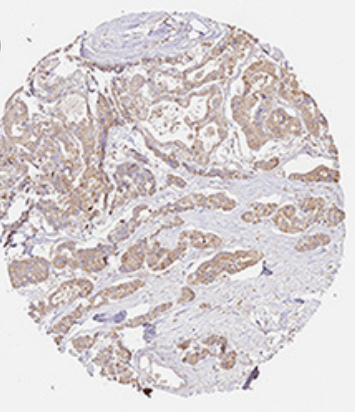

Medium
UBE2C

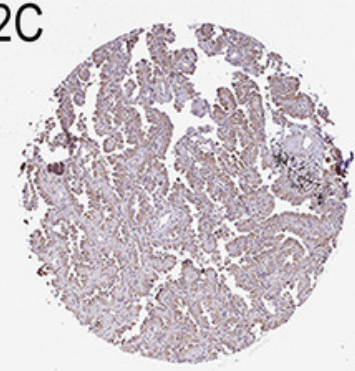

High 
Figure 8

ROC curves of hub genes in TCGA database.

The ROC curves of (A-I) CDKN3, MKI67, CEP55, SPAG5, AURKA, TOP2A, UBE2C, CHEK1, and BIRC5. ROC, receiver operating characteristic. 

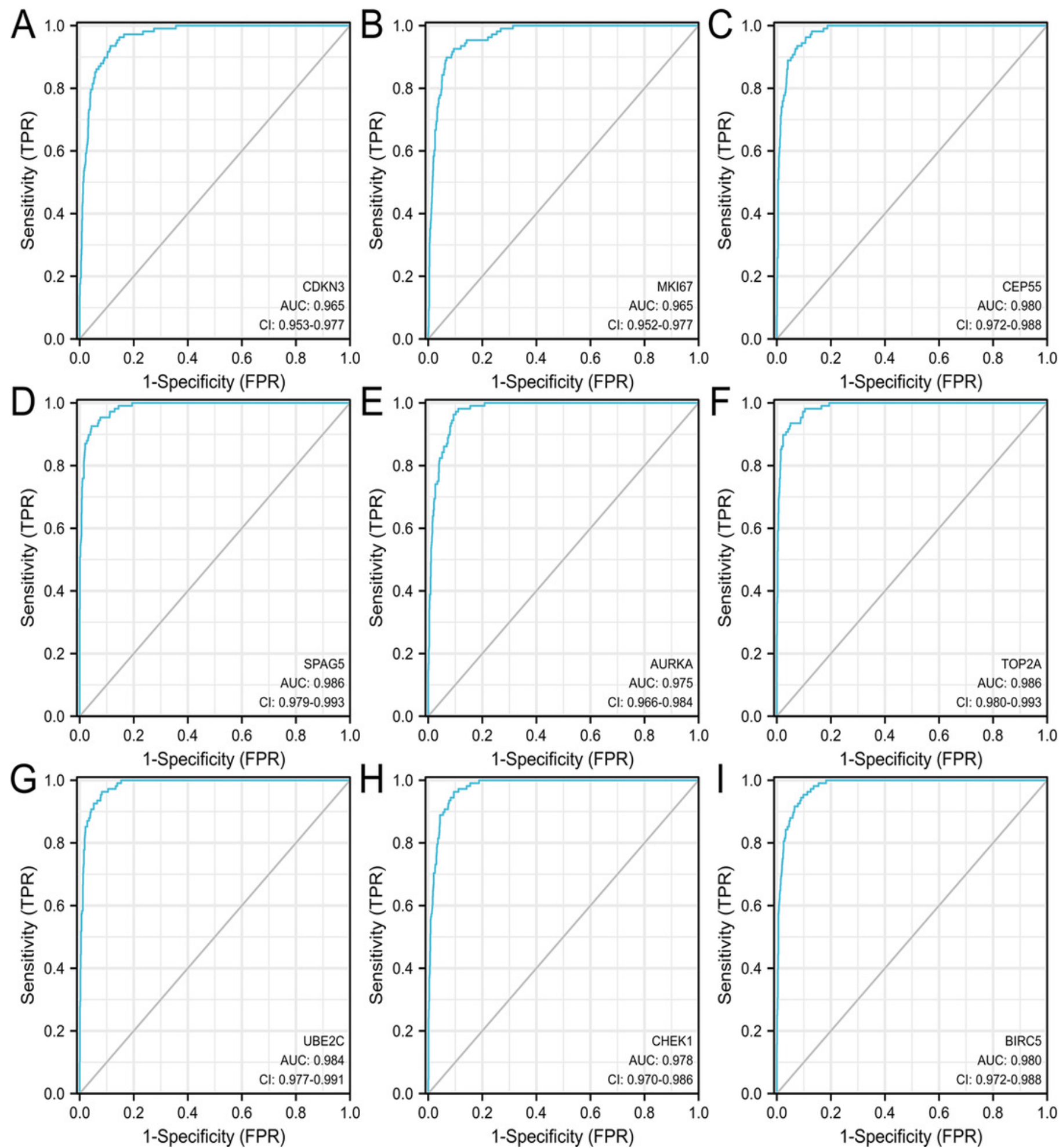
Figure 9

Correlation between the expression of (A-I) CDKN3, MKI67, CEP55, SPAG5, AURKA, TOP2A, UBE2C, CHEK1, BIRC5 and immune cell populations (B Cell, CD8+ T Cell, CD4+ T Cell, macrophage, neutrophil, and dendritic cell) in LUAD.

$P<0.05$ was considered statistically significant. 
A
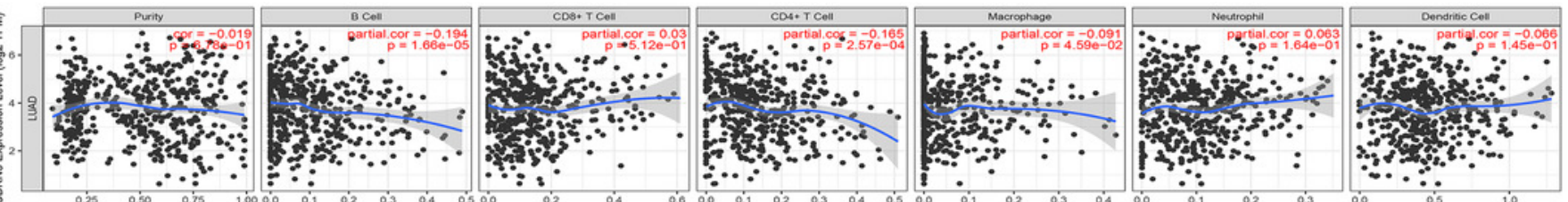

B
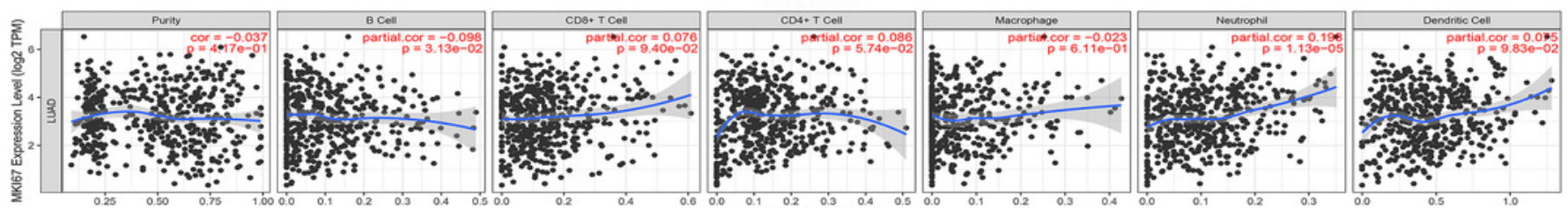

C
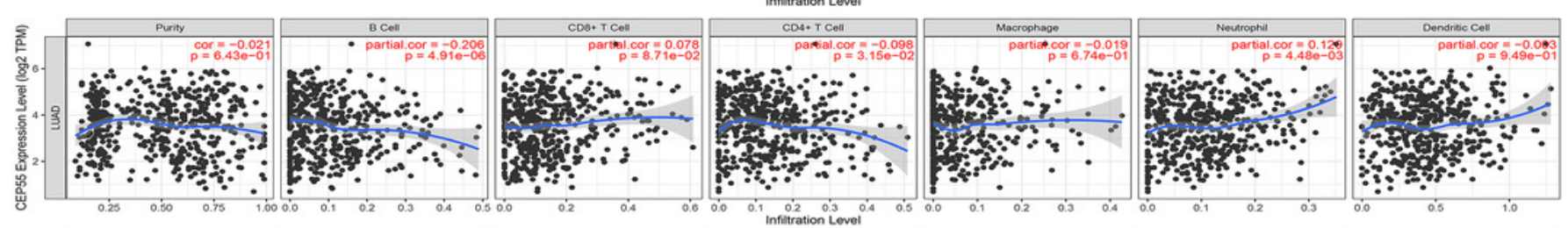

D
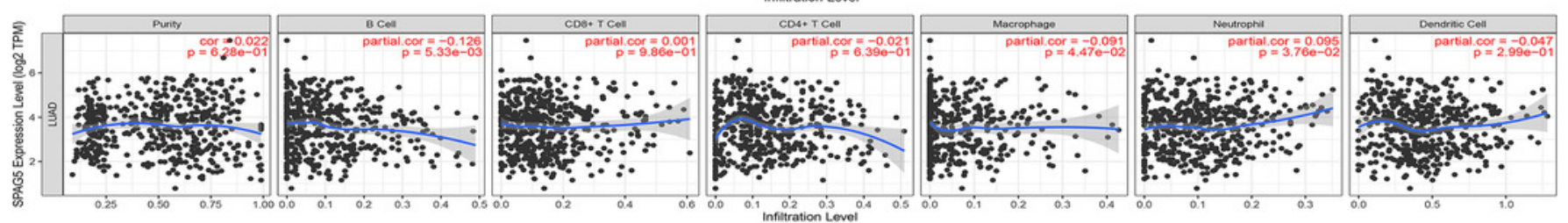

E
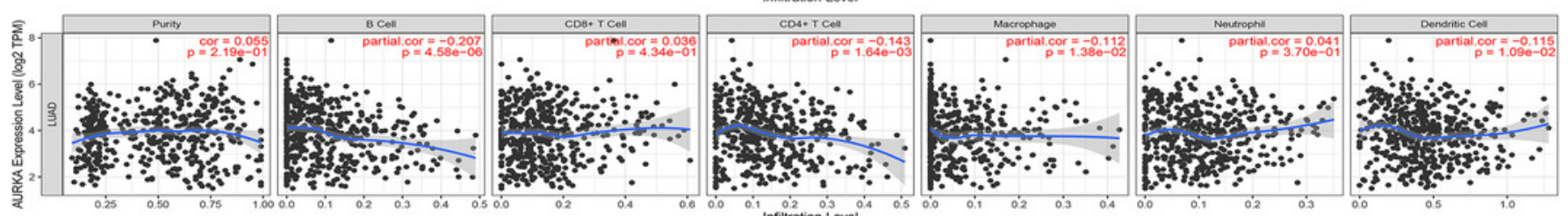

F
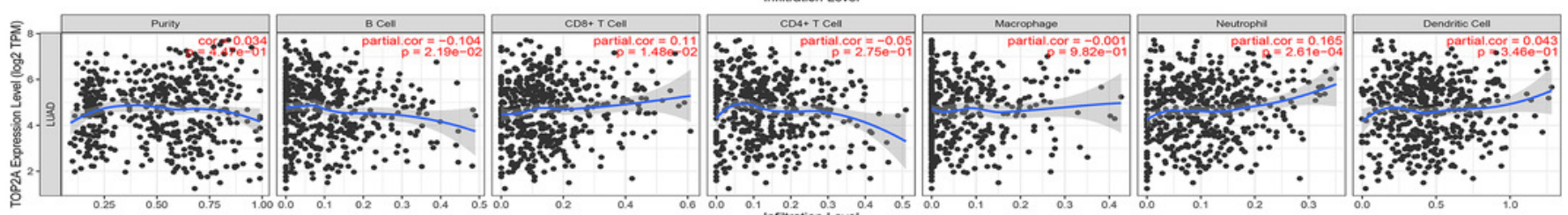

G
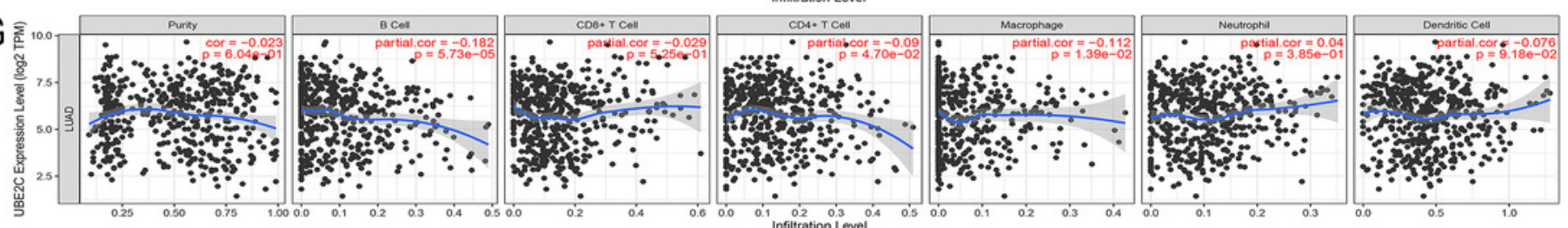

$\mathrm{H}$
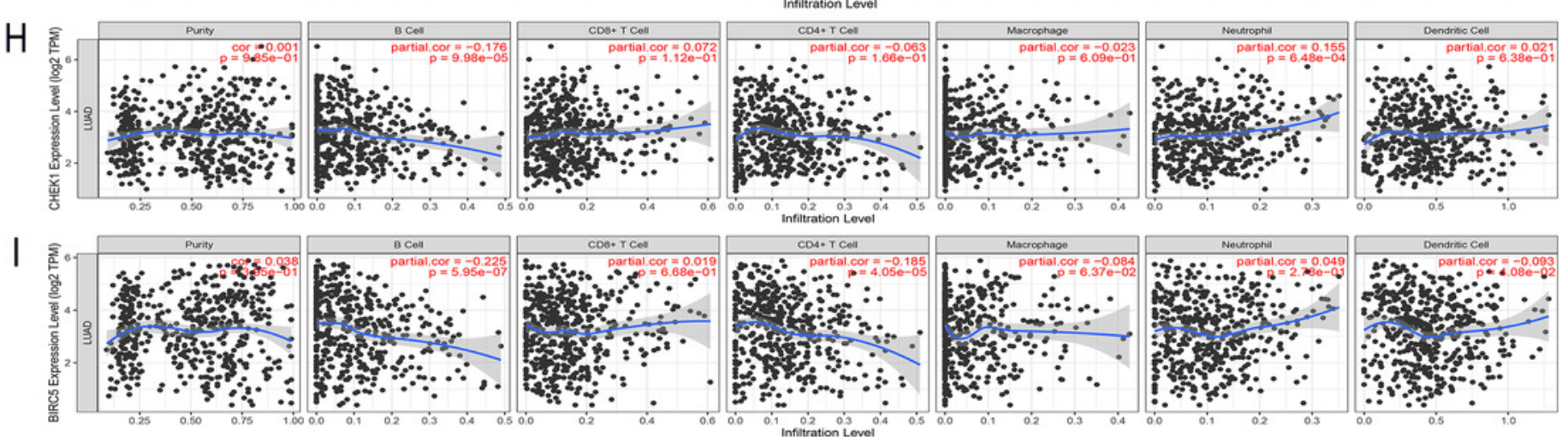
Figure 10

RT-PCR validation of the hub genes between lung cancer tissues and normal controls.

RT-PCR validation of the hub genes between lung cancer tissues and normal controls ( $* \mathrm{P}<$ $0.05, * * P<0.01)$.

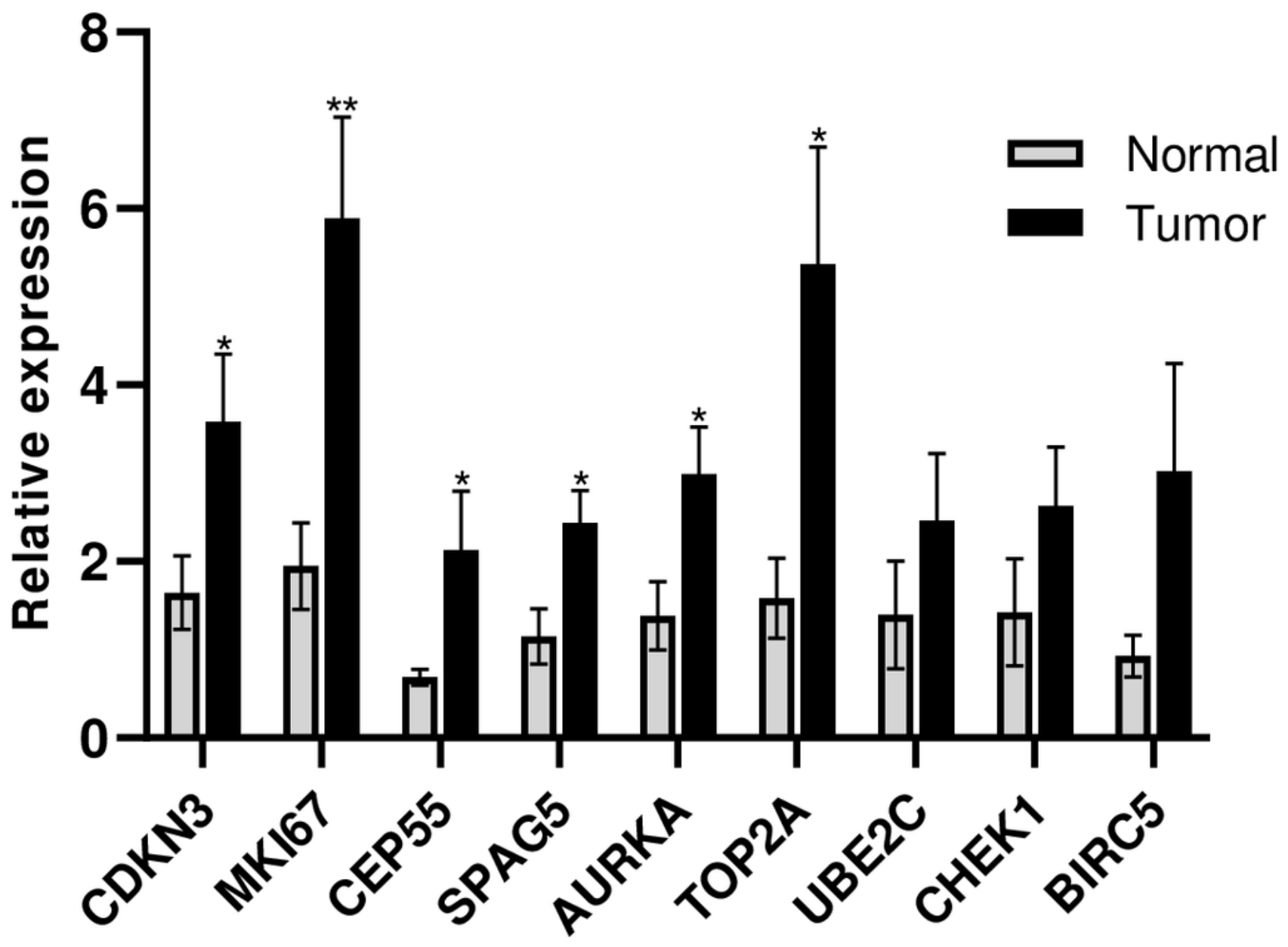




\section{Table 1 (on next page)}

Characteristics of the six GEO datasets. 
1 Table 1. Characteristics of the six GEO datasets.

\begin{tabular}{cccc}
\hline GEO ID & Platform & Normal samples (n) & Tumor samples(n) \\
\hline GSE136043 & GPL13497 & 5 & 5 \\
GSE130779 & GPL20115 & 8 & 8 \\
GSE118370 & GPL570 & 6 & 6 \\
GSE85841 & GPL20115 & 8 & 8 \\
GSE85716 & GPL19612 & 6 & 6 \\
GSE89039 & GPL17077 & 8 & 8 \\
\hline
\end{tabular}

2 GEO, Gene Expression Omnibus. 
Table 2 (on next page)

The scored top 20 genes in Degree, EPC, MCC, and MNC algorithms. 
1 Table 2. The scored top 20 genes in Degree, EPC, MCC, and MNC algorithms.

\begin{tabular}{ccccc}
\hline \multirow{2}{*}{ Category } & \multicolumn{4}{c}{ Rank methods in cytoHubba } \\
& Degree & EPC & MCC & MNC \\
\hline 1 & IL6 & IL6 & TOP2A & IL6 \\
2 & MMP9 & MMP9 & UBE2C & MMP9 \\
3 & PECAM1 & PECAM1 & CEP55 & PECAM1 \\
4 & COL1A1 & SPP1 & SPAG5 & COL1A1 \\
5 & UBE2C & COL1A1 & CENPF & BMP2 \\
6 & BMP2 & PPARG & KIF20A & PPARG \\
7 & PPARG & CTGF & MELK & UBE2C \\
8 & CAV1 & MKI67 & CHEK1 & TOP2A \\
9 & CTGF & CHEK1 & AURKA & MKI67 \\
10 & TOP2A & TOP2A & BIRC5 & CTGF \\
11 & MKI67 & UBE2C & MKI67 & CHEK1 \\
12 & AURKA & CDKN3 & CDKN3 & AURKA \\
13 & CEP55 & BIRC5 & KIF14 & SPP1 \\
14 & SPP1 & CEP55 & TRIP13 & CAV1 \\
15 & BIRC5 & BMP2 & RAD54L & BIRC5 \\
16 & SPAG5 & AURKA & CENPM & KIF20A \\
17 & CDKN3 & MELK & CDC25C & SPAG5 \\
18 & CHEK1 & CENPF & GTSE1 & CDKN3 \\
19 & CENPF & SPAG5 & MND1 & MELK \\
20 & KIF20A & CDKN2A & STIL & CEP55 \\
\hline
\end{tabular}

2 Degree, node connect degree; EPC, edge percolated component; MCC, maximal clique centrality; MNC, maximal neighborhood 3 component. 
Table 3 (on next page)

The top 15 enriched GO terms of genes in mudule 1. 
1 Table 3. The top 15 enriched GO terms of genes in mudule 1.

\begin{tabular}{clcc}
\hline Category & \multicolumn{1}{c}{ Term } & Count & PValue \\
\hline BP & cell division & 7 & 0.000002 \\
BP & mitotic nuclear division & 6 & 0.000009 \\
BP & G2/M transition of mitotic cell cycle & 5 & 0.000019 \\
BP & chromosome segregation & 4 & 0.000069 \\
BP & protein localization to centrosome & 3 & 0.000204 \\
CC & midbody & 8 & 0.000000 \\
CC & nucleoplasm & 13 & 0.000006 \\
CC & Gcentriole & 5 & 0.000006 \\
CC & spindle & 5 & 0.000008 \\
CC & chromosome, centromeric region & 4 & 0.000032 \\
MF & ATP binding & 10 & 0.000013 \\
MF & protein binding & 19 & 0.000075 \\
MF & protein kinase binding & 4 & 0.008149 \\
MF & protein C-terminus binding & 3 & 0.017525 \\
MF & microtubule binding & 3 & 0.022512 \\
\hline
\end{tabular}

$2 \mathrm{BP}$, biological process; $\mathrm{CC}$, cell component; MF, molecular function. 